\title{
Investigating cosmological GAN emulators using latent space interpolation
}

\author{
Andrius Tamosiunas ${ }^{\oplus},{ }^{1,2 \star}$ Hans A. Winther, ${ }^{3}$ Kazuya Koyama, ${ }^{2}$ David J. Bacon ${ }^{\oplus},{ }^{2}$ Robert C. Nichol ${ }^{2}$ \\ and Ben Mawdsley ${ }^{\circledR 2}$ \\ ${ }^{1}$ School of Physics and Astronomy, University of Nottingham, University Park, Nottingham NG7 2RD, UK \\ ${ }^{2}$ Institute of Cosmology and Gravitation, University of Portsmouth, Dennis Sciama Building, Burnaby Road, Portsmouth PO1 3FX, UK \\ ${ }^{3}$ Institute of Theoretical Astrophysics, University of Oslo, Svein Rosselands hus, Blindern campus Sem Saelandsvei, Oslo 13 0371, Norway
}

Accepted 2021 June 25. Received 2021 June 18; in original form 2020 November 20

\begin{abstract}
Generative adversarial networks (GANs) have been recently applied as a novel emulation technique for large-scale structure simulations. Recent results show that GANs can be used as a fast and efficient emulator for producing novel weak lensing convergence maps as well as cosmic web data in 2D and 3D. However, like any algorithm, the GAN approach comes with a set of limitations, such as an unstable training procedure, inherent randomness of the produced outputs, and difficulties when training the algorithm on multiple data sets. In this work, we employ a number of techniques commonly used in the machine learning literature to address the mentioned limitations. Specifically, we train a GAN to produce weak lensing convergence maps and dark matter overdensity field data for multiple redshifts, cosmological parameters, and modified gravity models. In addition, we train a GAN using the newest Illustris data to emulate dark matter, gas, and internal energy distribution data simultaneously. Finally, we apply the technique of latent space interpolation as a tool for understanding the feature space of the GAN algorithm. We show that the latent space interpolation procedure allows the generation of outputs with intermediate cosmological parameters that were not included in the training data. Our results indicate a 1-20 percent difference between the power spectra of the GAN-produced and the test data samples depending on the data set used and whether Gaussian smoothing was applied. Similarly, the Minkowski functional analysis indicates a good agreement between the emulated and the real images for most of the studied data sets.
\end{abstract}

Key words: hydrodynamics - methods: numerical - software: simulations - large-scale structure of Universe.

\section{INTRODUCTION}

In the era of precision cosmology an important tool for studying the evolution of large-scale structure is $N$-body simulations. Such simulations evolve a large number of particles under the influence of gravity (and possibly other forces) throughout cosmic time and allow detailed studies of the non-linear structure formation. Modern cosmological simulations are highly realistic and extremely complex and may include galaxy evolution, feedback processes, massive neutrinos, weak lensing, and many other effects. Such complexity, however, comes at a price in terms of computational resources and large simulations may take several days or even weeks to run. In addition, to fully account for galaxy formation and other effects various simplification schemes and semi-analytical models are required. To address these issues, a variety of emulation techniques have been discussed in the literature (Kwan et al. 2015; Knabenhans et al. 2019; Winther et al. 2019). In light of upcoming surveys like Euclid, such emulators will be an invaluable tool for producing mock data quickly and efficiently.

Lately, machine learning techniques have been explored as a valuable tool in cosmology, with applications ranging widely from cosmological parameter extraction from observational data to su-

^E-mail: andrius.tamosiunas@nottingham.ac.uk pernovae classification (Merten et al. 2019; Ntampaka et al. 2019). Machine learning techniques have also been applied as an alternative to the traditional emulation methods. For instance, deep learning has been used to accurately predict non-linear structure formation (He et al. 2019). Similarly, generative adversarial networks (GANs) and variational autoencoders (VAEs) have been used to produce novel realistic cosmic web 2D projections, weak lensing maps, and to perform dark energy model selection (Kingma \& Welling 2013; Goodfellow et al. 2014; Rodríguez et al. 2018; Li, Li \& Zhang 2019; Mustafa et al. 2019; Ullmo, Decelle \& Aghanim 2020). In addition, the GAN approach has also been used to produce realistic cosmic microwave background temperature anisotropy 2D patches as well as deep-field astronomical images (Mishra, Reddy \& Nigam 2019; Smith \& Geach 2019). Finally, generating full 3D cosmic web data has been discussed in Perraudin et al. (2019) and Kodi Ramanah et al. (2020). The cited works show that GANs are capable of reproducing a variety of cosmological simulation outputs efficiently and with high accuracy.

However, certain challenges remain: the training process of the GAN algorithm is complicated and prone to failure and producing full scale $3 \mathrm{D}$ results is computationally expensive. A common problem when training GANs is mode collapse, when the generator neural network overpowers the discriminator and gets stuck in producing a small sample of identical outputs. Mode collapse can be addressed in multiple ways - modern GAN architectures introduce label flipping 


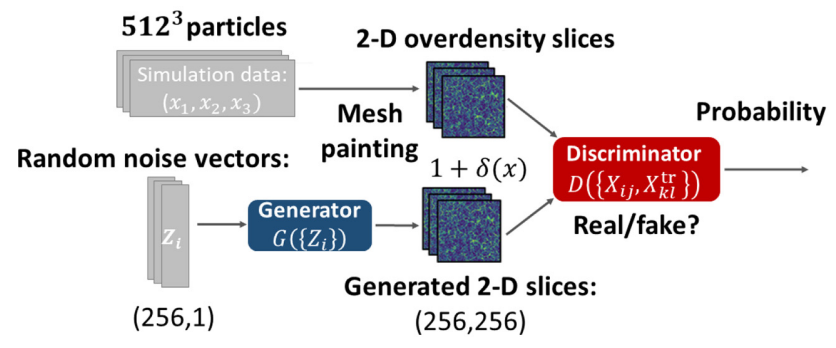

Figure 1. The pipeline of training a GAN on 2D DM-only cosmic web slices. A number of $512^{3}$ particle simulation boxes are mesh-painted and sliced to produce the $256 \times 256 \mathrm{px}$ cosmic web slice training data set. The training data set is then used to train the system of the generator and the discriminator neural networks. Once the training procedure is finished, the generator can be used to generate novel 2D cosmic web slices out of random Gaussian noise vectors. A nearly analogous procedure is used to train the weak lensing convergence maps, with the main difference being that an extra step of ray tracing is required in order to produce the training data set.

or use different loss functions, such as Wasserstein distance, which has been shown to reduce the probability of mode collapse (Arjovsky, Chintala \& Bottou 2017).

In addition, GANs and other neural network based approaches suffer from being black box algorithms - i.e. it is usually difficult to determine the specific features that are deemed important by the algorithm during the training procedure. This issue is of special importance when training GANs on scientific data as such information could hold important clues about the non-trivial correlations in the data set. For instance, a GAN trained on weak lensing convergence maps with different cosmological parameters could contain important information about the effects of cosmology on weak lensing that is not captured by the usual summary statistics.

In this paper, we address some of these issues and present our results on extending some of the currently existing GAN algorithms. In particular, we use a modified version of the cosmoGAN algorithm (introduced in Mustafa et al. 2019) to produce weak lensing convergence maps and 2D cosmic web projections of different redshifts and multiple cosmologies. In addition, the GAN is trained on dark matter, gas, and internal energy data simultaneously. Furthermore, we explore techniques from contemporary research in the field of deep learning, such as latent space interpolation, as a way to control the outputs of the algorithm and as a tool to explore the structure of the latent space. Finally, we apply the latent space interpolation techniques for producing data of unseen cosmological parameters.

Similar issues have been recently explored in Rodríguez et al. (2018), Mustafa et al. (2019), and Perraudin et al. (2020). In Perraudin et al. (2020) in particular, the authors employ a conditional GAN architecture to produce weak lensing convergence maps with different cosmological parameters (Mirza \& Osindero 2014). The key feature of the conditional GAN architecture is that in addition to the latent space vector input one adds a parameter vector, which allows one to specify the cosmological parameters directly. This feature allows direct control of the algorithm outputs. The main difference of our approach is that the DCGAN (Radford, Metz \& Chintala 2015) architecture described in this work does not allow direct control of the outputs. This introduces extra difficulties when training the GAN algorithm on data set that consists of data with multiple cosmological parameters. However, we explore multiple alternative approaches based on latent space interpolation. In doing so, we also explore the structure of the latent space produced during the training procedure, which is the first step towards tackling the black box problem of the GAN algorithm.

Finally, we discuss GANs in the framework of Riemannian geometry in order to put our problem on a more theoretical footing and to explore the feature space learnt by the algorithm.

\section{GENERATIVE ADVERSARIAL NETWORKS}

\subsection{The algorithm}

GANs, first introduced in a now seminal paper (Goodfellow et al. 2014), are a system of neural networks that are trained adversarially. In particular, a GAN consists of a generator - a neural network responsible for producing data from random noise and a discriminator, which is responsible for evaluating the produced data against the training set. The two neural networks compete in an adversarial fashion during the training process - the generator is optimized to produce realistic data sets statistically identical to the training data and hence to fool the discriminator. Mathematically, such an optimization corresponds to minimizing the following cost function:

$$
\begin{aligned}
\min _{G_{\phi}} \max _{D_{\theta}} J\left(D_{\theta}, G_{\phi}\right)= & -\mathbb{E}_{X \sim \mathbb{P}_{r}} \log \left(D_{\theta}(X)\right) \\
& -\mathbb{E}_{Z \sim \mathbb{P}_{g}} \log \left(1-D_{\theta}\left(G_{\phi}(Z)\right)\right),
\end{aligned}
$$

where $\mathbb{E}$ refers to the expectation function, $D_{\theta}$ refers to the discriminator with weights $\theta, G_{\phi}$ refers to the generator with weights $\phi$, $\mathbb{P}_{r}$ refers to the distribution of the data we are aiming for, $\mathbb{P}_{g}$ refers to the generated distribution, $X$ refers to the data (real or generated) analysed by the discriminator, and $Z$ refers to the random noise vector input to the generator.

Such an optimization procedure is a nice example of game theory where the two agents (the generator and the discriminator) compete in a two player zero sum game and adjust their strategies (neural network weights) based on the common cost function. In case of perfect convergence, the GAN would reach Nash equilibrium, i.e. the generator and the discriminator would reach optimal configurations (optimal sets of weights). In practice, however, reaching convergence is difficult and the training procedure is often unstable and prone to mode collapse (Farnia \& Ozdaglar 2020).

The two neural networks, the discriminator and the generator, have two different training procedures. In particular, the discriminator classifies the data sets into real (coming from the training data set) or fake (produced by the generator) and is penalized for misclassification via the discriminator loss term. The discriminator weights are updated through backpropagation as usual (Linnainmaa 1976; Rumelhart, Hinton \& Williams 1986). The generator, on the other hand, samples random noise, produces an image, gets the classification of that image from the discriminator and updates its weights accordingly via backpropagation using the generator loss function term. The full training procedure is done by alternating between the discriminator and the generator training cycles.

Assuming the adversarial training is successful, the generator $G_{\phi}(Z)$ can then be used separately for producing realistic synthetic data from a randomized input vectors $Z$. Fig. 1 lays out the pipeline for using a GAN to generate dark matter (DM)-only cosmic web slice data. ${ }^{1}$

\footnotetext{
${ }^{1} \mathrm{~A}$ note on the used terminology: cosmic web slices in this work refer to the 2D overdensity field projections generated by slicing full 3D overdensity field data from $N$-body simulations. Such slices are then used to train the GAN
} 


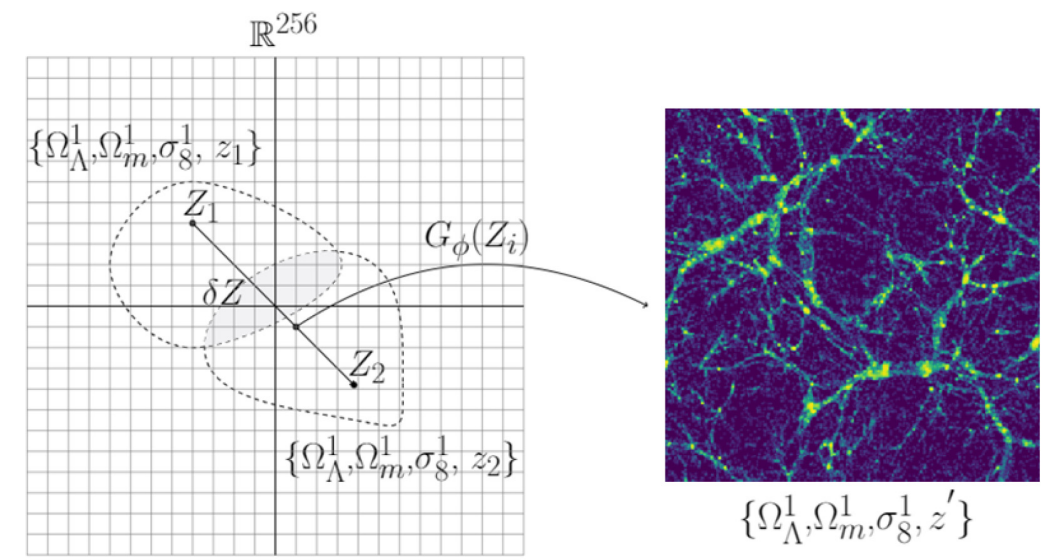

Figure 2. Illustration of the latent space interpolation procedure. Training the GAN algorithm on the cosmic web slices of two different redshifts encodes two different clusters in the latent space (which is a subset of a 256-dimensional space, i.e. the size of the random noise input vector). Sampling a point from the line connecting two input points $Z_{1}$ and $Z_{2}$ in this space produces an output with redshift $z^{\prime}$. As we will see, in the case of our data set with $z_{1}=1.0$ and $z_{2}=0.0$, several points near the centre of this line correspond to outputs approximately emulating $z^{\prime} \approx 0.5$.

\subsection{Latent space interpolation}

The generator neural network with its multilayered structure can be represented mathematically as a function composition:

$G_{\phi}\left(Z_{i}\right)=g^{1} \circ g^{2} \circ \ldots \circ g^{n} \quad$ with $\quad g_{k}^{i}\left(y^{i}\right)=S\left(W_{k}^{i} y^{i}+b^{i}\right)$,

where each layer $g^{i}$ maps from an input $y^{i}$ to an output as shown above. Here, $G_{\phi}\left(Z_{i}\right)$ is the generator neural network, $Z_{i}$ is the random input vector, $S(y)$ is a non-linear activation function, $W_{k}^{i}$ is the weight matrix, and $b^{i}$ is the bias term. The aim of the training procedure is to find an optimal weight matrix $W$ (along with the bias terms), which maps the input to the wanted output.

If the training procedure is successful, the generator $G_{\phi}\left(Z_{i}\right)$ learns to map the values of a random vector $Z_{i}$ to the values of a statistically realistic $2 \mathrm{D}$ array representing the output $X_{j k}$ (a cosmic web slice or a convergence map in our case). This can be viewed as mapping from a low-dimensional latent space $Z \subseteq \mathbb{R}^{d}$ to a higher dimensional data (pixel) space $X \subseteq \mathbb{R}^{D}$ (for more details, see Shao, Kumar \& Fletcher 2017 and Appendix B). For a generator neural network $d \ll D$ (in our case $d=256$ or 64 , while $D=256^{2}$ ).

The generator network has a number of interesting properties. In particular, during the training procedure it maps clusters in the $Z$ space to the clusters in the $X$ space. Hence, if we treat the random input vectors $Z_{i}$ as points in a $d$-dimensional space, we can interpolate between multiple input vectors and produce a transition between the corresponding outputs. In particular, if we choose two input vectors $Z_{1}$ and $Z_{2}$ and find a line connecting them, sampling intermediate input points $Z_{i}$ along that line leads to a set of outputs that correspond to an almost smooth transition between outputs $X_{1}$ and $X_{2}$. As an example, if we train the generator to produce cosmic web slices of two different redshifts, we can produce a set of outputs corresponding to a transition between those two redshifts by linearly interpolating between the input vectors $Z_{1}$ and $Z_{2}$ (see Fig. 2). More concretely, if we train the algorithm on cosmic web slices of redshifts $\{0.0$, $1.0\}$, somewhere between the two input vectors, one can find a point $Z^{\prime}$, which produces an output that has a matter power spectrum approximately corresponding to a redshift $z^{\prime} \approx 0.5$ (see Fig. 17 and Appendix D). This is fascinating given that the training data set did not include intermediate redshift data. Here, it is important to note that such an interpolation procedure does not necessarily produce a perfectly smooth transition in the data space, i.e. the produced outputs corresponding to the latent space vectors $Z_{i}$ between $Z_{1}$ and $Z_{2}$ are not always realistic (in terms of the matter power spectrum and other statistics; see Fig. 18 and Section 5.6 for further details). Also, one might naively think that the point $Z^{\prime}$ lies in the middle of the line connecting $Z_{1}$ and $Z_{2}$ but in general we found it not to be the case (as the middle of the mentioned line does not necessary correspond to the middle between $X_{1}$ and $X_{2}$ in the data space, which is known to be non-Euclidean; see Appendix B). In this work, we investigate whether the latent space interpolation procedure can be used to map between outputs of different redshifts and cosmologies and whether the produced data sets are physically realistic.

The latent space interpolation technique was performed by randomly choosing two input vectors $Z_{1}$ and $Z_{2}$, finding the line connecting the two points in the 256 (64)-dimensional space (256 (64) is the size of $Z_{1}$ and $Z_{2}$ ) and then sampling 64 equally spaced points along that line. The outputs of the generator neural network of these intermediate input points $G_{\phi}\left(Z_{\text {int }}\right)$ then correspond to cosmic web slices and weak lensing maps that represent a transition between the two outputs $G_{\phi}\left(Z_{1}\right)$ and $G_{\phi}\left(Z_{2}\right)$.

In order to perform linear latent space interpolation, it is crucial to have the ability to distinguish between different data classes produced by the GAN (e.g. cosmic web slices of different redshifts). To resolve this problem, we employed a combination of the usual summary statistics like the power spectrum and the Minkowski functionals along with a machine learning algorithm. In particular, we tested using gradient boosted decision trees for distinguishing the different classes of data sets produced by the GAN (Chen \& Guestrin 2016; see Section 5.6 for a further discussion of the chosen approach).

\section{DATA SETS AND THE TRAINING PROCEDURE}

\subsection{Weak lensing convergence map data}

Gravitational potentials influence the path of photons in such a way that they introduce coherent distortions in the apparent shape (shear) and position of light sources. Weak gravitional lensing introduces ellipticity changes in objects of the order of $\approx 1$ percent and can be measured across the sky, meaning that maps of the lensing distortion of objects can be made and related to maps of the mass distribution in the Universe. The magnitude of the shear depends 
upon the combined effect of the gravitational potentials between the source and the observer. An observer will detect this integrated effect and maps of the integrated mass, or convergence, can be made. Gravitational lensing has the significant advantage that it is sensitive to both luminous and dark matter, and can therefore directly detect the combined matter distribution. In addition, weak lensing convergence maps allow for detecting the growth of structure in the Universe and hence they can also be used for probing statistics beyond twopoint correlation functions, such as in the higher moments of the convergence field or by observing the topology of the field with Minkowski functionals and peak statistics (Dietrich \& Hartlap 2010; Mawdsley et al. 2020). As future surveys attempt to further probe the non-linear regime of structure growth, the information held in these higher order statistics will become increasingly important, and will also require accurate simulations in order to provide cosmological constraints. This requirement for large numbers of simulations that also model complex physical phenomena means that more computationally efficient alternatives to $\mathrm{N}$-body simulations, such as the GAN approach proposed in this work, are required.

In order to train the GAN algorithm to produce realistic convergence maps, we used publicly available data sets. In particular, to test whether we can reproduce the original results from Mustafa et al. (2019) we used the publicly available data from Mustafa et al. (2020). The data set consisted of 8000 weak lensing maps that were originally produced by running a GADGET2 (Springel 2005) simulation with $512^{3}$ particles in $240 \mathrm{Mpc} h^{-1}$ box. To perform ray tracing, the GADGET weak lensing simulation pipeline was used. The simulation box was rotated multiple times for each ray tracing procedure, resulting in $100012 \mathrm{deg}^{2}$ maps per simulation box.

In order to train the GAN algorithm on convergence maps of different cosmologies and redshifts, we used the publicly available Columbia Lensing data set described in Zorrilla Matilla et al. (2016), Gupta et al. (2018), and Columbia Lensing (2020). The available data set contains weak lensing convergence maps covering a field of view of $3.5 \mathrm{deg} \times 3.5 \mathrm{deg}$, with resolution of $1024 \times 1024$ pixels. The maps were originally produced using GADGET2 DMonly simulation data with $240 \mathrm{Mpc} h^{-1}$ side cube and $512^{3}$ particles. The data set includes 96 different cosmologies (with varying $\Omega_{\mathrm{m}}$ and $\sigma_{8}$ parameters). The values of $\Omega_{\mathrm{m}}=0.260$ and $\sigma_{8}=0.8$ were used as the fiducial cosmology. In our analysis, we only used a small subset of this data set, namely, the maps where only one of the two cosmological parameter varies. In particular, we worked with the data set consisting of the maps with $\sigma_{8}=\{0.436,0.814\}$ with a common value of $\Omega_{\mathrm{m}}=0.233$. This was done in order to simplify the latent space analysis.

For the weak lensing map data, we used the same architecture as described in table 1 in Rodríguez et al. (2018). In fact the same basic architecture with minor variations was used for training all the data sets described later on (see Tables $\mathrm{C} 1$ and $\mathrm{C} 2$ ). In particular, for the cosmic web slice data we increased the random input vector size to 256 (from 64 in the case of weak lensing maps). For the multicomponent data set (dark matter, gas, and internal energy), we changed the input layer to account for the 3D input data. In summary, for the discriminator, we used an architecture of four convolutional layers with batch normalization and LeakyRelu activation (Fukushima 1980; Lecun et al. 1998; Nair \& Hinton 2010; Maas 2013; Ioffe \& Szegedy 2015). In the final layer, we used the sigmoid activation. For the generator, we used a linear input layer followed by four deconvolution layers with Relu activation and batch normalization. The output layer used the tanh activation function. The key parameter in terms of the training procedure is the learning rate. For all the cosmic web slice data sets, we found the learning rate value of $R_{L}=3 \times 10^{-5}$ to work well. In the case of all the considered weak lensing data sets we used $R_{L}=9 \times 10^{-6}$. The training procedure and all the key parameters are described in great detail in the publicly available code (see Section 6 for more information).

\subsection{Cosmic web slice data}

The cosmic web or the dark matter overdensity field refers to the intricate network of filaments and voids as seen in the output data of $\mathrm{N}$-body simulations. The statistical features of the cosmic web contain important information about the underlying cosmology and could hide imprints of modifications to the standard laws of gravity. In addition, emulating a large number of overdensity fields is important for reliable estimation of the errors of cosmological parameters. Hence, emulators, such as the one proposed in this work, will be of special importance for the statistical analysis in the context of the upcoming observational surveys.

To build the cosmic web training data set, we used a similar procedure to the one outlined in Rodríguez et al. (2018). In particular, we ran L-PICOLA (Howlett, Manera \& Percival 2015) to produce a total of 15 independent simulation boxes with different cosmologies. Initially, we used the same cosmology as described in Rodríguez et al. (2018) with $h=0.7, \Omega_{\Lambda}=0.72$, and $\Omega_{\mathrm{m}}=0.28$. Subsequently, we studied the effects of varying one of the cosmological parameters, namely the $\sigma_{8}$ parameter. We explored the values of $\sigma_{8}=\{0.7,0.8$, $0.9\}$ along with $\Omega_{\Lambda}=0.7, \Omega_{\mathrm{m}}=0.3$, and $h=0.67$. For each different set of simulations, we saved snapshots at three different redshifts: $z=\{0.0,0.5,1.0\}$. For each simulation, we used a box size of 512 $\mathrm{Mpc} h^{-1}$ and a number of particles of $512^{3}$. For the latent space interpolation procedure, we trained the GAN on slices with redshifts $\{0.0,1.0\}$, with a common value of $\sigma_{8}=0.8$.

To produce the slices for training the GAN, we used nbodykit (Hand et al. 2018), which allows painting an overdensity field from a catalogue of simulated particles. To obtain the needed slices, we cut the simulation box into sections of $2 \mathrm{Mpc}$ width in $x, y, z$ directions and for each section a mesh painting procedure was done. This refers to splitting the section into cells, where the numerical value of each cell corresponds to the dark matter overdensity $1+\delta(x)$. Finally, after a $2 \mathrm{D}$ projection of each slice, a $256^{2}$ px image was obtained, with each pixel value corresponding to the overdensity field. To emphasize the features of the large-scale structure, we applied the same nonlinear transformation as described in Rodríguez et al. (2018): $s(x)=$ $2 x /(x+a)-1$, with $a=250$, which rescales the overdensity values to $[-1,1]$ and increases the contrast of the images.

In order to emulate modified gravity effects, we used the MGPICOLA code, which extends the original L-PICOLA code in order to allow simulating theories that exhibit scale-dependent growth (Scoccimarro et al. 2012; Tassev, Zaldarriaga \& Eisenstein 2013; Winther et al. 2017; Winther 2020). This includes models, such as $f(R)$ theories that replace the Ricci scalar with a more general function in the Einstein-Hilbert action (see Li \& Koyama 2019 for an overview of the phenomenology of such models). In particular, multiple runs of MG-PICOLA were run with the following range of the $f_{R 0}$ parameter: $\left[10^{-7}, 10^{-1}\right]$. Such a wide range was chosen to make the latent space interpolation procedure easier. The $f(R)$ simulations were also run with the same seed as the corresponding $\Lambda \mathrm{CDM}$ simulations, making the two data sets described above directly comparable.

\subsection{Dark matter, gas, and internal energy data}

Simultaneously generating the dark matter and the corresponding baryonic overdensity field data is a great challenge from both the 


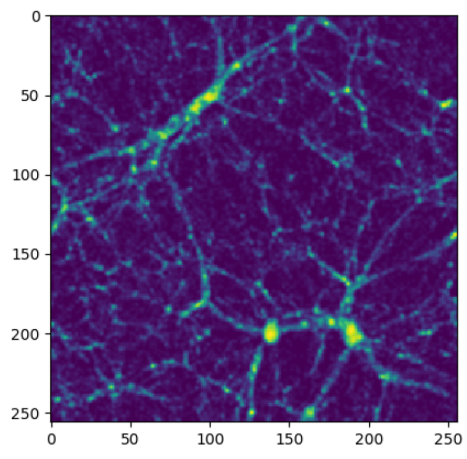

(a) DM overdensity field

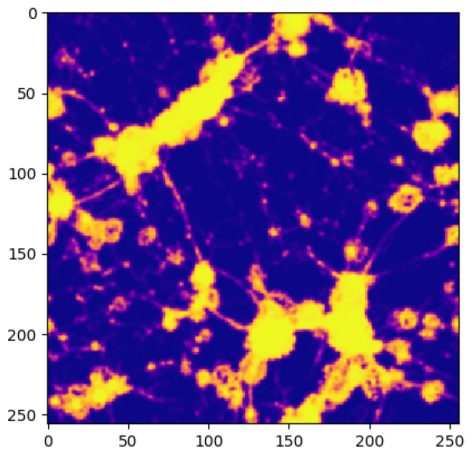

(c) Internal energy field

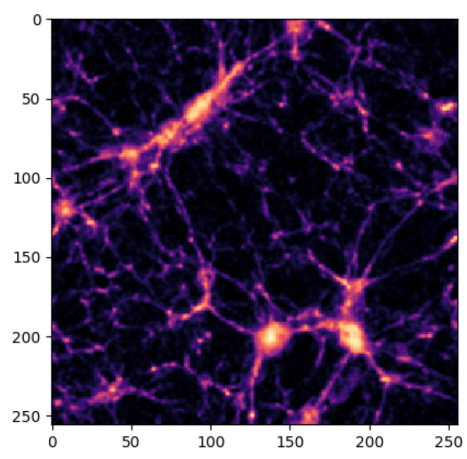

(b) Gas overdensity field

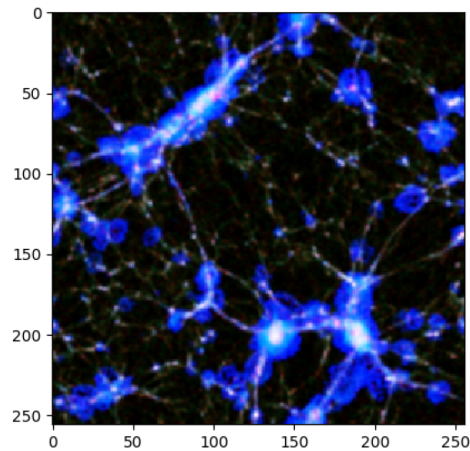

(d) All components combined

Figure 3. Samples from the Illustris simulation data set used to train the GAN algorithm: 2D slices of the different simulation components.

theoretical and the computational perspectives. Namely, generating the baryonic distribution requires detailed hydrodynamical simulations that account for the intricacies of galaxy formation and feedback processes, which leads to a major increase in the required computational resources. For this reason, emulating large amounts of hydrodynamical simulation data is of special importance.

To produce the dark matter, baryonic matter, and the internal energy distribution slices, we used the publicly available Illustris3 simulation data (Vogelsberger et al. 2014; Nelson et al. 2015). Illustris-3 refers to the low-resolution Illustris run including the fullphysics model with a box size of $75000 \mathrm{kpc}^{-1}$ and over $9 \times 10^{7}$ dark matter and gas tracer particles. The cosmology of the simulation can be summarized by the following parameters: $\Omega_{\mathrm{m}}=0.2726, \Omega_{\Lambda}=$ $0.7274, h=0.704$. The simulation included the following physical effects: radiative gas cooling, star formation, galactic-scale winds from star formation feedback, supermassive blackhole formation, accretion, and feedback.

To form the training data set, we used an analogous procedure to the one used for the cosmic web slices in Section 3.2. In particular, we sliced the full simulation box into slices of $100 \mathrm{kpc} h^{-1}$ and for each slice used mesh painting to obtain an overdensity field. This was done for the dark matter and gas data. In addition, we also used the available internal energy (thermal energy in the units of $(\mathrm{km} / \mathrm{s})^{2}$ ) distribution data. Fig. 3 shows a few samples from the data set.

To investigate whether the GAN algorithm could be trained on multidimensional array data, we treated the DM, gas, and energy distribution 2D slices as red giant branch (RGB) planes in a single image. In particular, a common way of representing colours in an image is forming a full colour image out of three planes, each corresponding to the pixel values for red, green, and blue colours. In this framework, a full-colour image corresponds to a 3D array. Convolutional neural networks, including the one that the cosmoGAN algorithm is based on, are originally designed to be trained on such RGB images. Hence, we combined the mentioned DM, gas, and internal energy slices into a set of RGB arrays that were used as a training set.

\subsection{The training procedure}

The initial stages of training (i.e. reproducing the results in Rodríguez et al. 2018 and Mustafa et al. 2019) were done using the Google Cloud computing platform. The following set-up was used: 4 standard vCPUs with 15 GB memory, 1 NVIDIA Tesla K80 GPU, and 2TB of SSD hard drive space. We found the typical training time using this set-up to be around $48 \mathrm{~h}$. This corresponds to an estimated $\mathrm{CO}_{2}$ emission of $8.93 \mathrm{~kg} \mathrm{CO}_{2}$ equation of which 100 per cent were directly offset by the cloud provider (Lacoste et al. 2019).

The later stages of training (i.e. training the GAN on different cosmology, modified gravity, and redshift data) were done using the local Sciama HPC cluster, which has 3702 cores of $2.66 \mathrm{GHz}$ Intel Xeon processors with 2 GB of memory per core. The training procedure was found to be at least five times slower when compared to the outlined Google Cloud set-up, as the HPC did not have access to a functional GPU and an older version of Tensorflow as used.

It is important to note that the GAN training procedure is significantly longer than running a small conventional simulation (e.g. a few hours per L-PICOLA simulation). However, the advantage of GANs lies in the fact that once the training is done, thousands of novel samples can be produced in a matter of seconds.

Given how unstable the GAN training procedure is we used a simple procedure of evaluating the best checkpoint: we calculated the mean square difference between the mean values of the GANproduced and the test data set power spectra, pixel histograms, and 

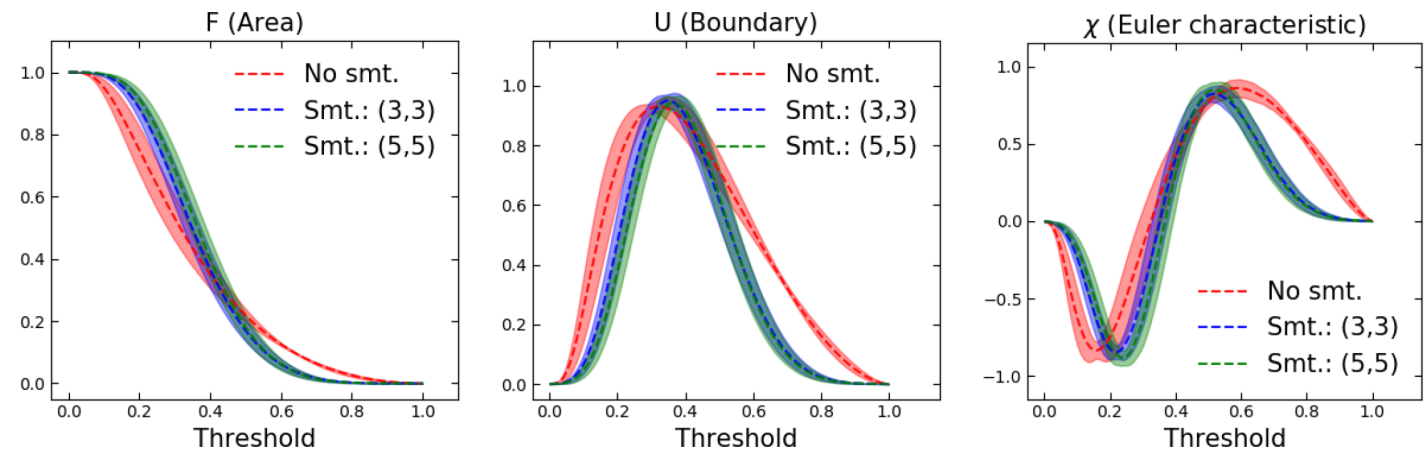

Figure 4. An illustration of the effects of Gaussian smoothing on the Minkowski functionals calculated using cosmic web slices from the training data with redshift $z=0.0$. The coloured bands correspond to the mean and the standard deviation of the functionals calculated using different sizes of Gaussian smoothing kernels on a batch of 64 images. Smt. refers to Gaussian smoothing with the numbers in the brackets corresponding to the kernel size.

the Minkowski functionals. The set of GAN weights that minimize this value was used for the plots displayed in the result section.

\section{DIAGNOSTICS}

The results produced by the algorithm were investigated using the following diagnostics: the 2D matter power spectrum, overdensity (pixel) value histogram, and the three Minkowski functionals. In addition, we computed the cross and the auto power spectrum in order to investigate the correlations between the data sets on different scales. The cross power spectrum was calculated using

$\left\langle\overline{\delta_{1}}(l) \overline{\delta_{2}^{*}}\left(l^{\prime}\right)\right\rangle=(2 \pi)^{2} \delta_{D}\left(l-l^{\prime}\right) P_{\times}(l)$,

where $\overline{\delta_{1}}$ and $\overline{\delta_{2}^{*}}$ are the Fourier transforms of the two overdensity fields at some Fourier bin $l$ and $\delta_{D}$ is the Dirac delta function.

The Minkowski functionals are a useful tool in studying the morphological features of fields that provide not only the information of spatial correlations but also the information on object shapes and topology. For some field $f(x)$ in $2 \mathrm{D}$ space, we can define the three Minkowski functionals as follows:

$V_{0}(v)=\int_{Q_{v}} \mathrm{~d} \Omega, \quad V_{1}(v)=\int_{\partial Q_{v}} \frac{1}{4} \mathrm{~d} l, \quad V_{2}(v)=\int_{\partial Q_{v}} \frac{\kappa_{c} \mathrm{~d} l}{2 \pi}$,

where $Q_{\nu} \equiv\left\{x \in \mathbb{R}^{2} \mid f(x)>v\right\}$ is the area and $\partial Q_{\nu} \equiv\{x \in$ $\left.\mathbb{R}^{2} \mid f(x)=v\right\}$ is the boundary of the field above threshold value $v$. The integrals $V_{0}, V_{1}$, and $V_{2}$ correspond to the area, boundary length, and the integrated geodesic curvature $\kappa_{c}$ along the boundary. In simple words, the procedure of measuring the Minkowski functionals refers to taking the values of the field at and above a given threshold $v$, evaluating the integrals in equation (4) and then changing the threshold for a range of values.

Minkowski fuctionals are a useful tool in weak lensing convergence map studies as they allow us to capture non-Gaussian information on the small scales, which is not fully accessed by the power spectrum alone. In addition, Minkowski functionals have been used to detect different cosmologies, modified gravity models, and the effects of massive neutrinos in weak lensing convergence maps (Petri et al. 2013; Ling et al. 2015; Marques et al. 2019). Given the usefulness of Minkowski functionals in accessing the non-Gaussian information on the small scales, we chose to apply the functionals for studying the produced cosmic web projections as well. To calculate the Minkowski functionals properly on a 2D grid, we used the minkfncts $2 d$ algorithm, which utilizes a marching square algorithm as well as pixel weighting to capture the boundary lengths correctly (Mantz, Jacobs \& Mecke 2008; minkfncts2d Python package 2020).

Minkowski functionals are sensitive to the Gaussian smoothing applied to the GAN-produced images and the training data. Hence, it is important to study the effects of Gaussian smoothing as it might give a deeper insight into the detected differences between the data sets. The procedure of smoothing refers to a convolution between a chosen kernel and the pixels of an image. In more detail, a chosen kernel matrix is centred on each pixel of an image and each surounding pixel is multiplied by the values of the kernel and subsequently summed. In the simplest case, such a procedure corresponds to averaging a chosen number of pixels in a given image. In the case of Gaussian filtering, a Gaussian kernel is used instead.

To filter the noise, we used Gaussian smoothing with a $3 \times 3$ kernel window and a standard deviation of $1 \mathrm{px}$. We found the Minkowski functionals to be especially sensitive to any kind of smoothing. For instance, the position and the shape of the trough of the third Minkowski functional is highly sensitive to existence of any smallscale noise. Fig. 4 illustrates the effects of Gaussian smoothing with different kernel sizes on the three Minkowski functionals.

In addition to the outlined diagnostics, we also tested the uniqueness of the GAN-produced samples. More specifically, in order to show that the GAN is indeed producing novel data rather than just mimicking the training data set, we compared the GAN-produced samples with the training samples. A $1000 \mathrm{GAN}$-generated samples were compared against each sample in the training data set by calculating the difference in the power spectra, pixel intensity histogram, and the Minkowski functionals. For all the relevant data sets, it was found that the GAN algorithm was indeed producing novel data rather than just mimicking the training data, which agrees well with the previous results found in Mustafa et al. (2019).

\section{RESULTS}

\subsection{Weak lensing map results}

After around 150 epochs (corresponding to around $120 \mathrm{~h}$ on our HPC), the GAN started producing statistically realistic convergence maps as measured by the power spectrum and the Minkowski functionals. The diagnostics were computed at an ensemble level 100 batches of 64 convergence maps were produced by the GAN and the mean values along with the standard deviation were computed and compared against the training and the test (simulation data not used in the training procedure) data sets. An analogous procedure was done when calculating the pixel intensity distribution histograms. 


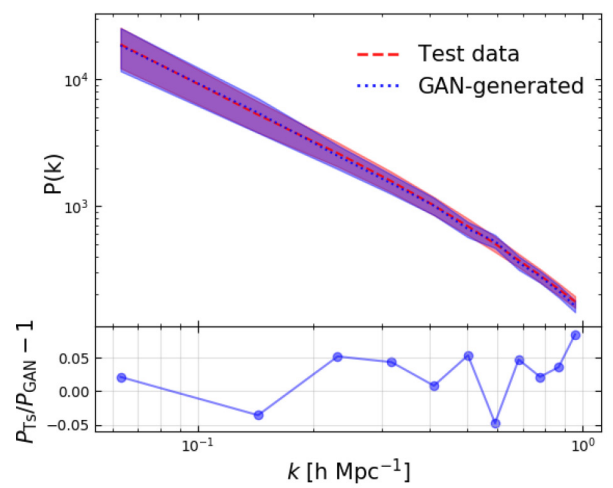

(a) Power spectrum

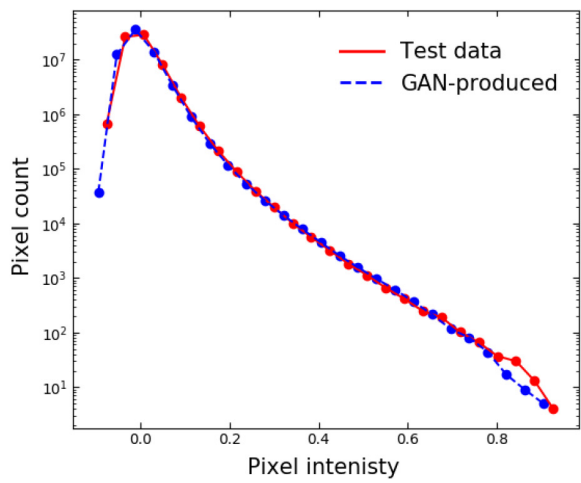

(b) Pixel intensity histogram

Figure 5. The matter power spectrum (with the relative difference) and the pixel intensity histogram for an ensemble of 6400 weak lensing convergence maps. The dashed lines correspond to the mean values, while the contours correspond to the standard deviation. Note that the pixel intensity values were normalized to the range of $[-1,1]$.
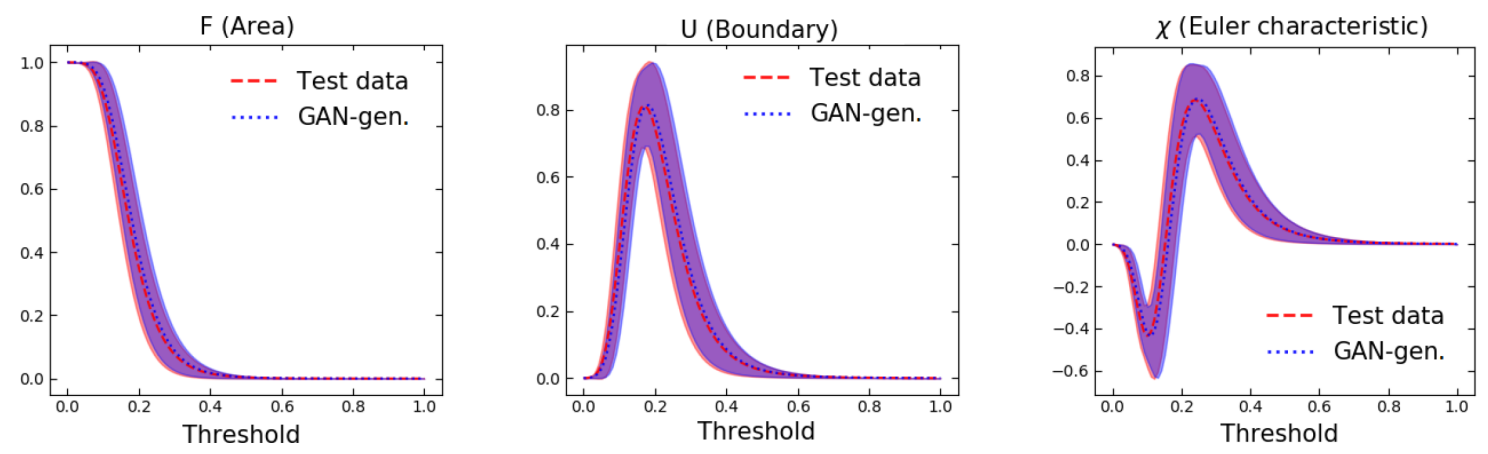

Figure 6. A comparison of the Minkowski functionals evaluated using 100 batches of 64 randomly selected maps for both data sets.

The two mentioned data sets were obtained by splitting the total available simulation data into $2 / 3$ dedicated to the training data set, while the other $1 / 3$ of the data was used as the test data set. The same general set-up was used when testing all the other data sets.

The power spectra agree well between the GAN-produced and the training and test data, with minor differences on the small scales (see Fig. 5). In particular, the difference between the test and the GANproduced data set power spectra is around 5 percent or lower for most values of $k$. Only at the smallest scales, a significant difference of 10 percent is reached. Similarly, the pixel intensity histogram in general shows a good agreement with significant differences appearing only for the highest and the lowest pixel intensity values (which is also detected in the original work in Mustafa et al. 2019). A selection of GAN-produced maps are presented for visual inspection in Fig. A1.

We also computed the Minkowski functionals for the GANproduced and the test data sets. The results are shown in Fig. 6. In general, there is a good agreement between the test data and the GAN-produced maps, given the standard deviation, however, some minor differences can be detected in the Euler characteristic and the boundary functional, likely resulting from noise.

\subsection{Weak lensing maps of multiple cosmologies}

We also found that the GAN is capable of producing realistic weak lensing maps for multiple cosmologies. This is an important result as it shows that the algorithm is able to pick up on the various subtle statistical differences between different cosmologies that usually requires a detailed study of the power spectrum, Minkowski functionals, and other statistics.

However, we found the training procedure to be highly prone to mode collapse. A wide hyperparameter search had to be performed to find an optimal set of parameters that did not lead to full or partial mode collapse. The most important parameter in this context was found to be the learning rate. As a rule of thumb, decreasing the learning rate led to mode collapse happening later in the training procedure. When the learning rate was reduced below a certain value (discussed further in the analysis section), mode collapse was avoided altogether. As in the case with the cosmic web slice data, applying a transformation to each pixel of the image in order to increase the contrast had a positive effect in reducing the probability of mode collapse as well.

Fig. 7 summarizes the results of training the GAN on shear maps with different $\sigma_{8}$ values. The results indicate an agreement of the power spectra in the range of 5-10 per cent for $k>10^{-1} h \mathrm{Mpc}^{-1}$ for $\sigma_{8}=0.814$. In the case of $\sigma_{8}=0.436$ the agreement is significantly better, ranging between 1 and 3 per cent on most scales. Interestingly, Gaussian smoothing increases the difference to around 5-15 per cent in this particular case. This shows that for this data set, Gaussian noise is not the major source of the statistical differences between the training and the GAN-generated data sets.

Fig. 8 compares the Minkowski functionals calculated using the training and the GAN-produced data sets. Given the standard 

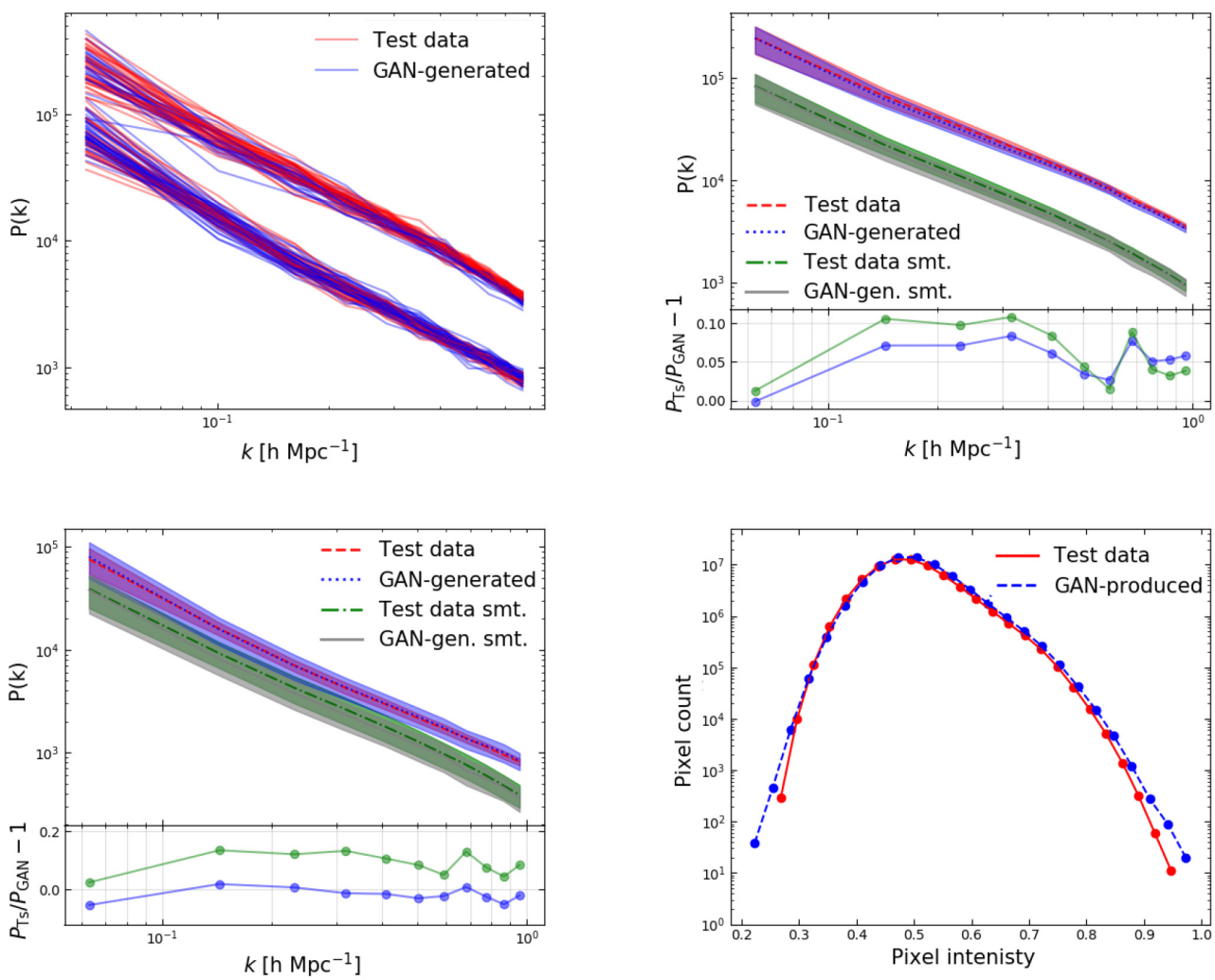

Figure 7. A selection of diagnostics to compare the test and the GAN-produced weak lensing convergence maps for $\sigma_{8}=\{0.436,0.814\}$ with $\Omega_{\mathrm{m}}=0.233$. Top left: Power spectra for an ensemble of 64 randomly chosen shear maps; top right: power spectra (mean and standard deviation) with and without Gaussian smoothing produced using 1000 randomly chosen shear maps with $\sigma_{8}=0.814$; bottom left: same as top right, but for $\sigma_{8}=0.436$; bottom right: the pixel intensity distribution (for both data sets combined). The blue and the green dots give $P_{\mathrm{Ts}} / P_{\mathrm{GAN}}-1$ with and without Gaussian smoothing applied correspondingly.
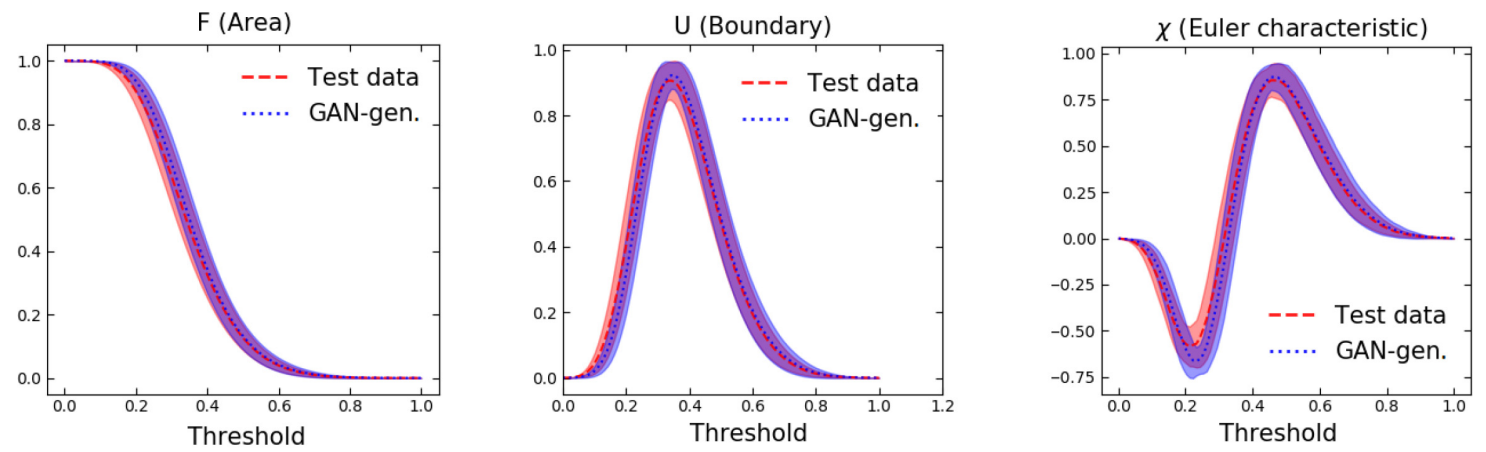

Figure 8. A comparison of the Minkowski functionals evaluated using 1000 randomly selected weak lensing convergence maps with $\sigma_{8}=\{0.436,0.814\}$. Gaussian smoothing is applied for all data sets.

deviation in both data sets, the results overlap for all threshold values. However, for thresholds in the range of $[0.0,0.4]$ there is a significant difference between the training and the GAN-generated data sets. We found that this is partially due to small-scale noise in the GAN-produced data (see Fig. 4). However, after experimenting with adding artificial noise to the training data set images, it is clear that the noise alone cannot fully account for the observed differences in the Minkowski functionals. Another reason for the observed differences could be a relatively small size of the used data set consisting of a few thousand weak lensing maps. It is likely that having more training data samples could significantly improve the results.

\subsection{Cosmic web for multiple redshifts}

We also found that the GAN is capable of producing realistic cosmic web $2 \mathrm{D}$ projections for different redshifts. As before with the weak lensing maps of different cosmologies, this result illustrates that the algorithm in general does not get confused between the two different redshifts and is capable of detecting subtle statistical differences between the different data sets (Fig. 9). In addition, we found that using Gaussian smoothing, as before, led to a better agreement between the training and the GAN-produced data sets. The effect is especially noticeable in the Minkowski functional analysis (Fig. 10). Visual samples of the produced cosmic web slices are shown in Fig. A2. 

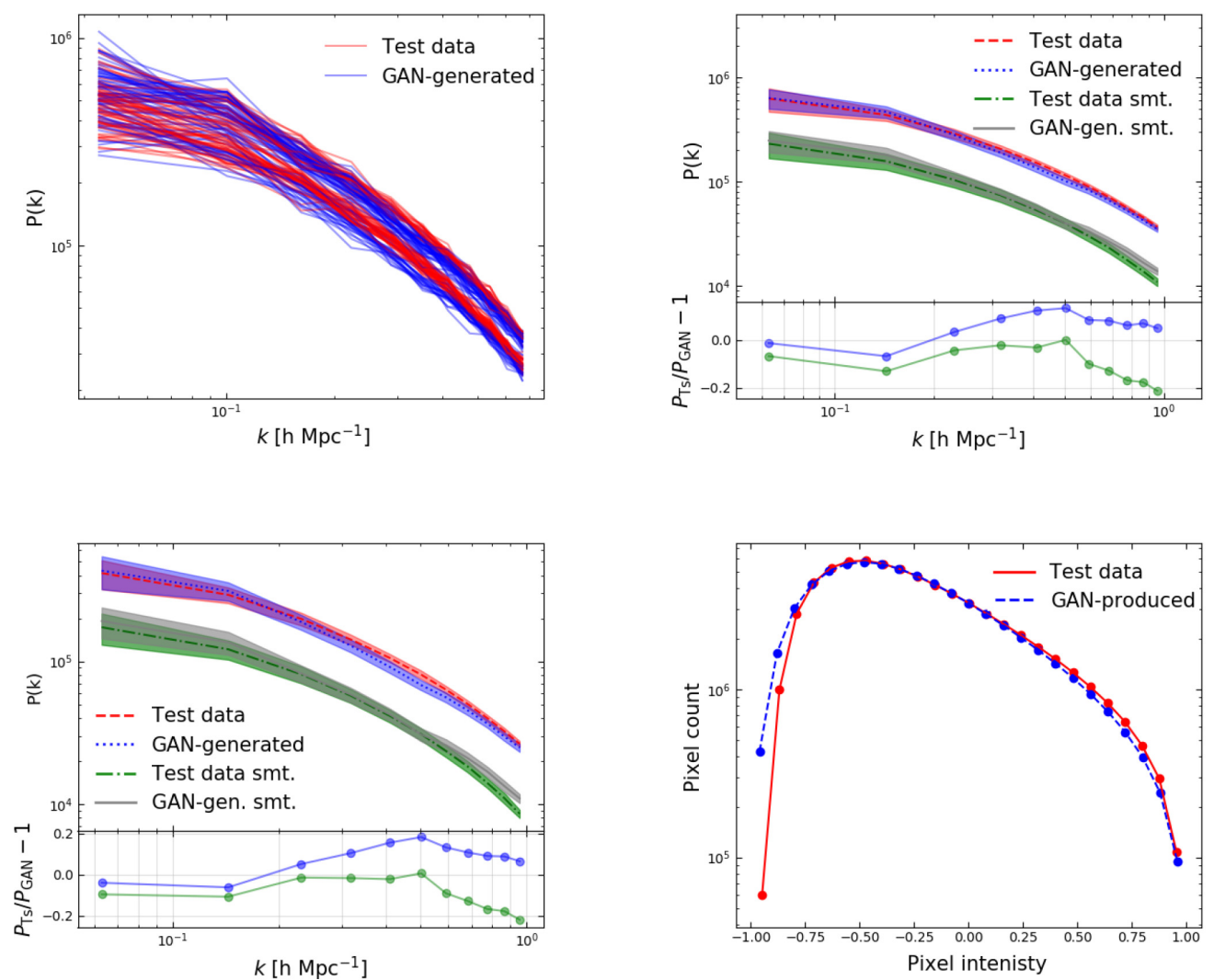

Figure 9. A selection of diagnostics to compare the test and the GAN-produced cosmic web slices for redshifts $z=0.0$ and $z=1.0$ with $\sigma_{8}=0.8$. Top left: Power spectra for an ensemble of 64 randomly chosen slices for two different redshifts; top right: mean and standard deviation of the power spectra produced using 1000 randomly chosen slices with $z=0.0$; bottom left: same as top right but for $z=1.0$; bottom right: the overdensity histogram (no smoothing). The blue and the green dots give $P_{\mathrm{Ts}} / P_{\mathrm{GAN}}-1$ with and without Gaussian smoothing applied correspondingly.
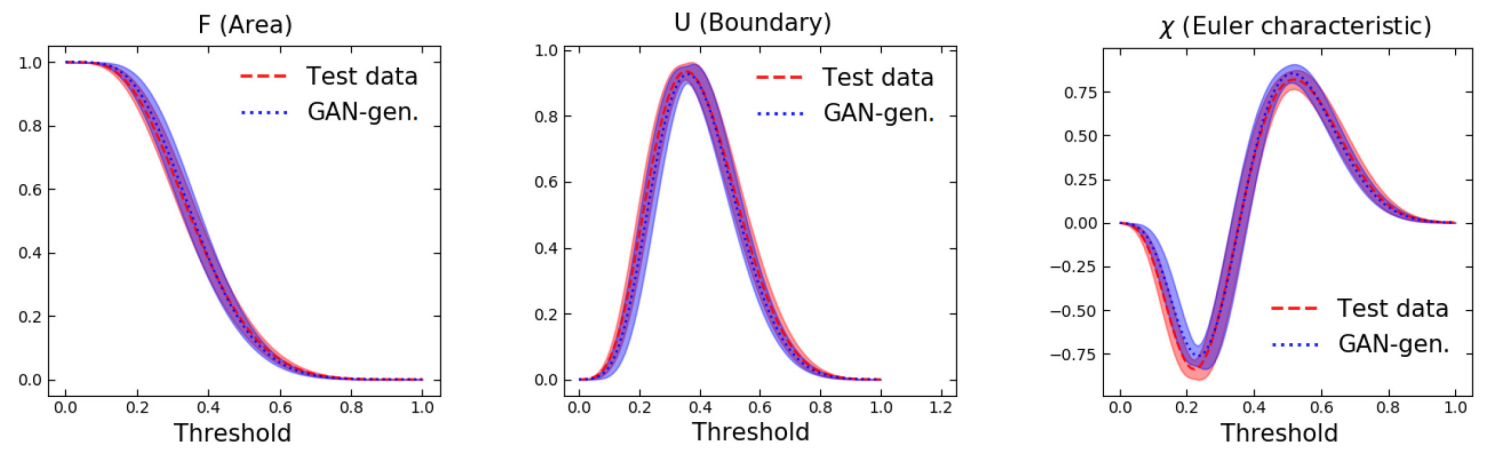

Figure 10. A comparison of the Minkowski functionals evaluated using 1000 randomly selected cosmic web slices of redshifts $z=\{0.0,1.0\}$ for both data sets. Gaussian smoothing is applied for all data sets.

We found the power spectra results for both redshift values to be very similar. Namely, for the non-smoothed case the difference between the test and the GAN-produced power spectra ranges between 5 and 10 per cent. The results are similar for the smoothed case, with exception of $k$ values around $1 h \mathrm{Mpc}^{-1}$ where the difference reaches 20 per cent.

The effects of the Gaussian smoothing on both the power spectra and the Minkowski functionals illustrate that one of the reasons for the differences between the GAN-generated and the test data sets is noise appearing on different scales in the GAN-produced images. Applying Gaussian smoothing, in general, filters the majority of such noise, however, it cannot fully account for all the differences appear- ing in the different statistical diagnostics. In addition, smoothing can improve the results on some scales, while worsening them on others. As an example, in Fig. 9, Gaussian smoothing increases the difference between the GAN-produced and the test data set power spectra on the smallest scales.

\subsection{Cosmic web for multiple cosmologies and modified gravity models}

Training the GAN on the cosmic web slices of different cosmologies and modified gravity models offered another way of testing whether the algorithm would pick up on the subtle statistical differences 

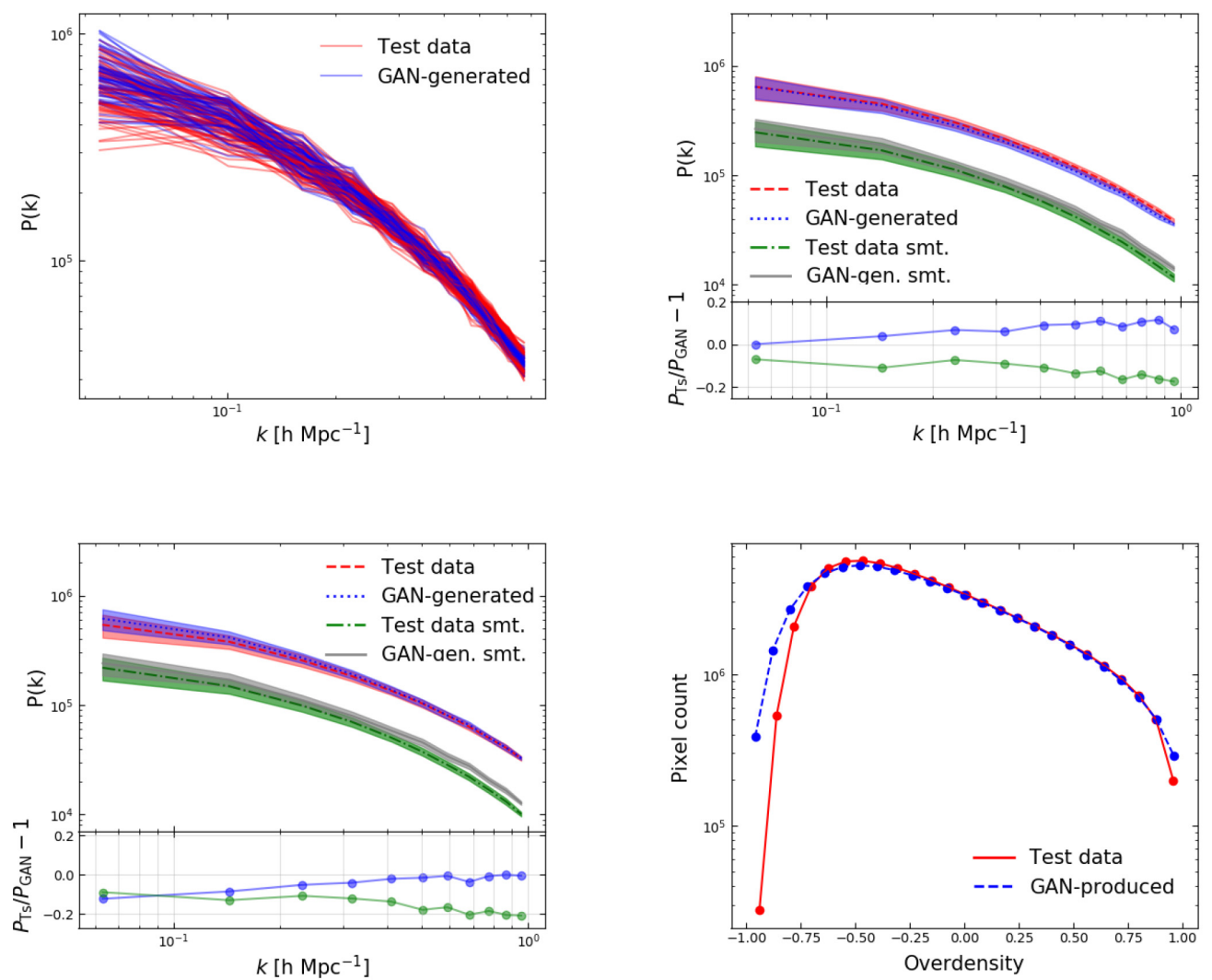

Figure 11. A selection of diagnostics to compare the test and the GAN-produced cosmic web slices for $\sigma_{8}=0.7$ and $\sigma_{8}=0.9$ at $z=0.0$. Top left: Power spectra for an ensemble of 64 randomly chosen slices for both data sets; top right: mean and standard deviation of the power spectra computed using 1000 randomly chosen slices of $\sigma_{8}=0.9$; bottom left: same as top right but for $\sigma_{8}=0.7$; bottom right: the overdensity histogram (no smoothing). The blue and the green dots give $P_{\mathrm{Ts}} / P_{\mathrm{GAN}}-1$ with and without Gaussian smoothing applied correspondingly.
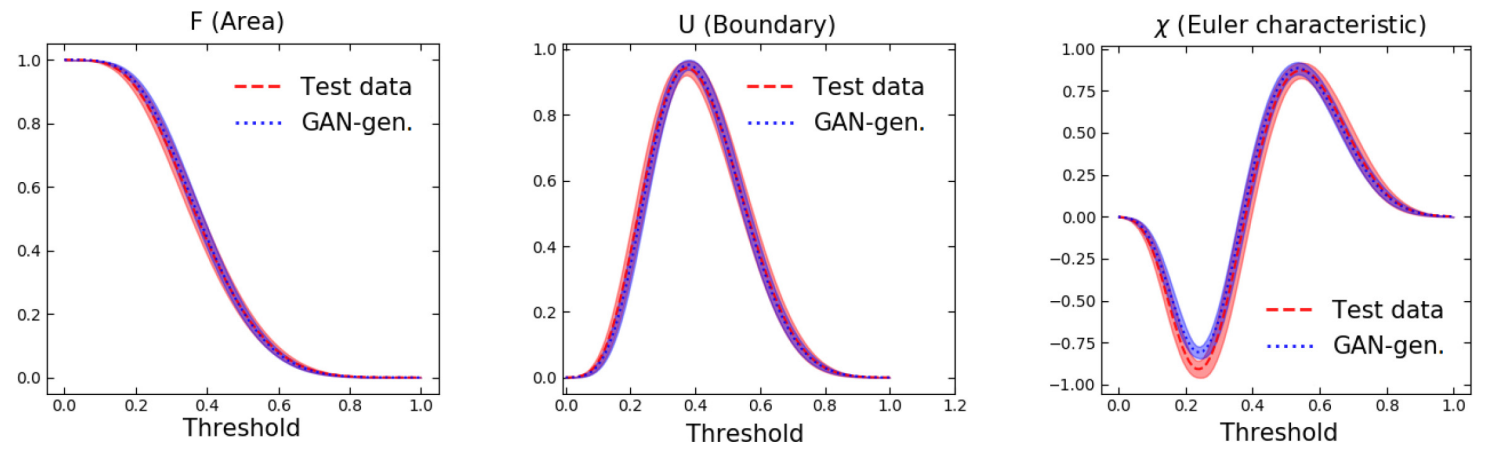

Figure 12. A comparison of the Minkowski functionals evaluated using 1000 randomly selected cosmic web slices from the data set with two different values of $\sigma_{8}=\{0.7,0.9\}$. Gaussian smoothing is applied for both data sets.

between the different data sets. In addition, the classification task for the discriminator neural network is more difficult when training on data sets with multiple cosmologies leading to longer training times.

The results indicate that the GAN is indeed capable of producing statistically realistic cosmic web data of different cosmologies and modified gravity models. With no Gaussian smoothing applied, the relative agreement between the power spectra is $1-10$ percent (see Fig. 11). Applying smoothing in this case resulted in increasing the relative power spectrum difference to over 10 per cent on average. In the case of cosmic web slices for different $f_{R 0}$ values, the agreement between the two data sets was good, ranging between 1 and 10 per cent on all scales. Smoothing improved the situation only in the mid-range of the covered $k$ values, reducing the agreement on the smallest scales .

Fig. 12 shows the Minkowski functional analysis. In this case, very little deviation is observed. In general, there is a good agreement between the GAN-produced and the training and the test data sets, especially for the first and the second Minkowski functionals. For the third Minkowski functional, the results diverge around the lower trough area, which is also observed for other data sets. This is at least in part related to small-scale noise as indicated by the previous analysis.

The results are similar for the GAN trained on cosmic web slices corresponding to different $f(R)$ models (Fig. 13 and Fig. 14). In general, we found a good agreement between the data sets, given 

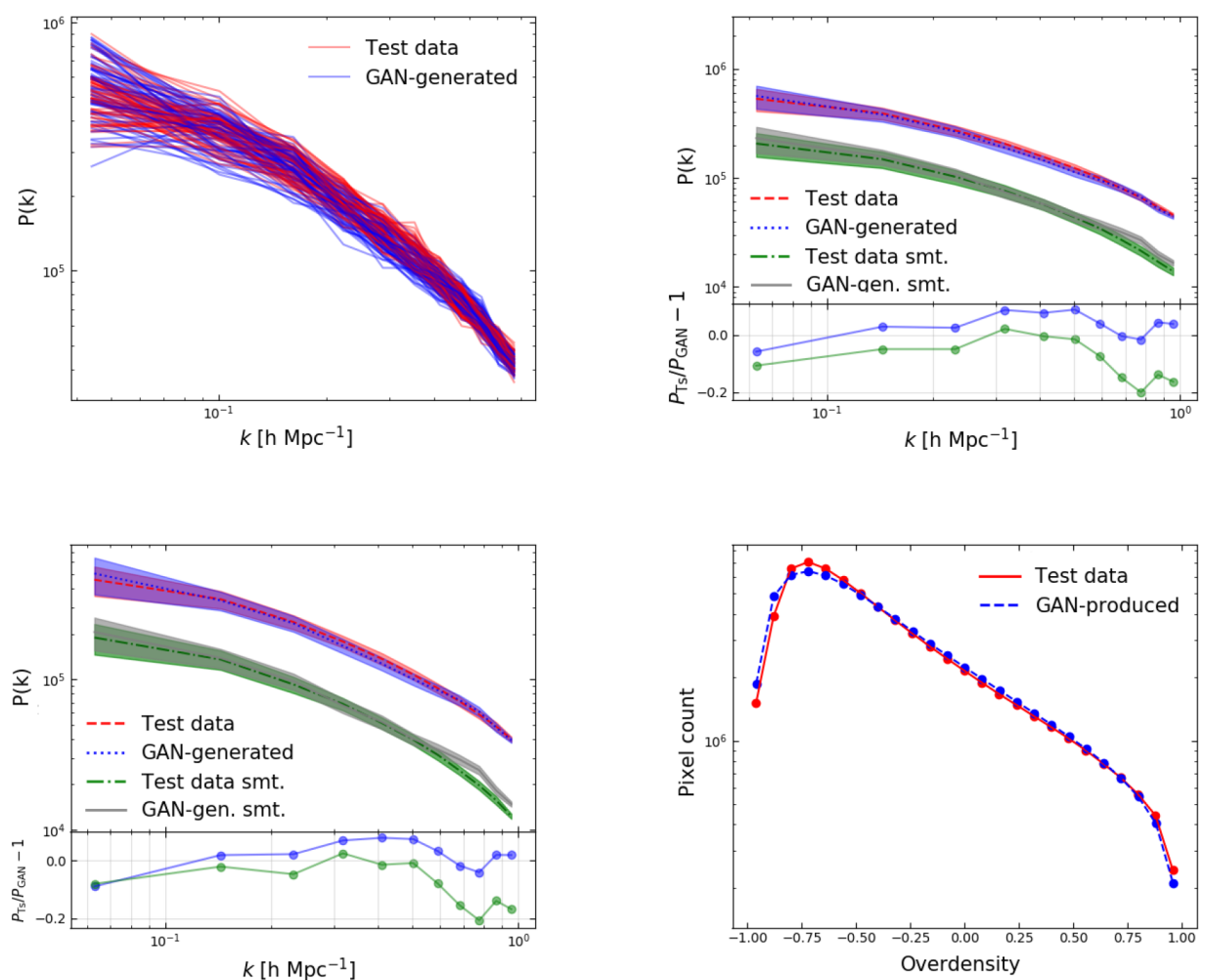

Figure 13. A selection of diagnostics to compare the test and the GAN-produced cosmic web slices for $f_{R 0}=\left\{10^{-7}, 10^{-1}\right\}$ (with $\sigma_{8}=0.8$ and $z=0.0$ ). Top left: Power spectra for an ensemble of 64 randomly chosen slices for both data sets; top right: mean and standard deviation of the power spectra produced using 1000 randomly chosen slices with $f_{R 0}=10^{-1}$; bottom left: same as top right but for $f_{R 0}=10^{-7}$; bottom right: the overdensity histogram (no smoothing). The blue and the green dots give $P_{\mathrm{Ts}} / P_{\mathrm{GAN}}-1$ with and without Gaussian smoothing applied correspondingly.
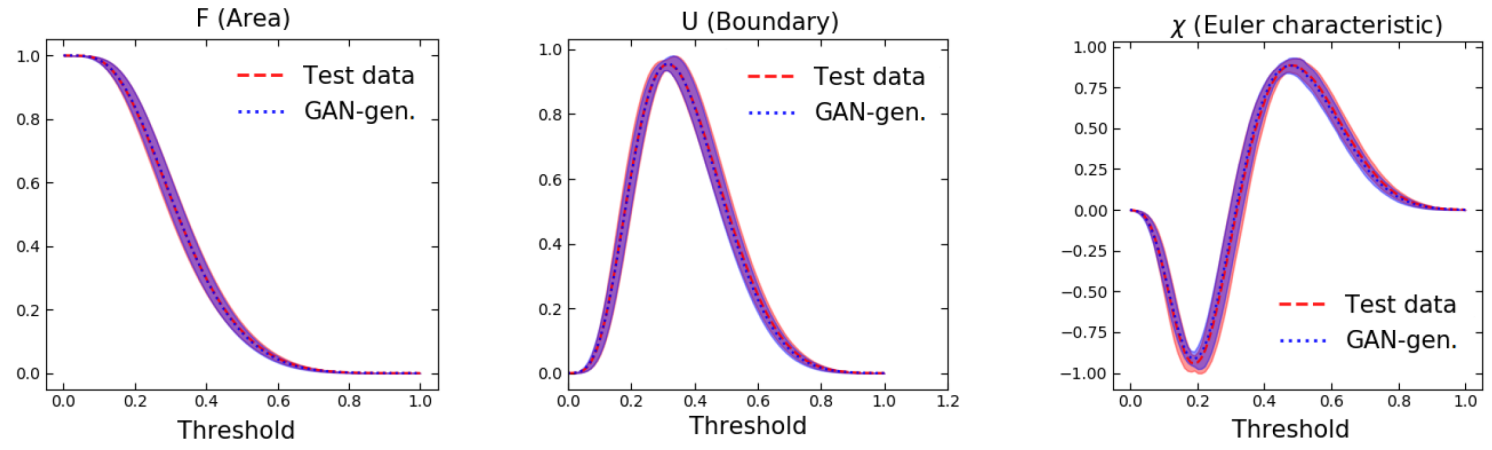

Figure 14. A comparison of the Minkowski functionals evaluated using 1000 randomly selected cosmic web slices from the data set with two different values of $f_{R 0}=\left\{10^{-7}, 10^{-1}\right\}$. Gaussian smoothing is applied for both data sets.

the standard deviation of the data and the GAN-produced results. Gaussian smoothing, in this case, was more effective in reducing some of the offset observed in the power spectrum analysis. However, it increased the offset on the smallest scales.

\subsection{Dark matter, gas, and internal energy results}

In the case of training the GAN algorithm on multiple components at the same time, we found the training procedure to be relatively quick and efficient (around 1.3 time quicker compared to the data sets discussed previously) despite the training data set being three times bigger. This is most likely due to the fact that the cosmic web slices in this particular data set corresponded to a much larger simulation box and hence were not as detailed on the smallest scales.
As before, we calculated the relative difference between the GAN-produced and the training and test data sets. The internal energy slices were analysed using Minkowski functionals as well as the cross power spectrum (Fig. 15). The analysis was done for both dark matter and the gas components. The relative difference between the power spectra for both DM and gas cosmic web slices was found to be at around 5 percent level for all the covered range. Gaussian smoothing reduced this value to 15 percent. In addition, the cross power spectrum was calculated for all the components. For both the dark matter-gas and the gasenergy pairs, there is a good agreement between the test and the GAN-produced data sets given the large standard deviation. Both plots show values well above zero for most $k$ values indicating a significant correlation between the dark matter and the corresponding 

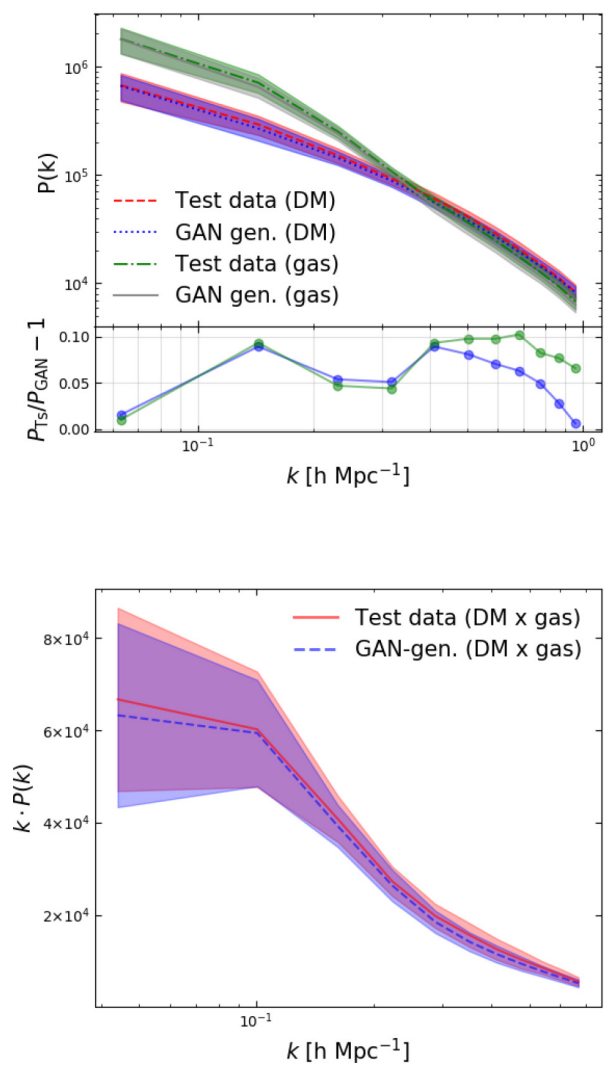
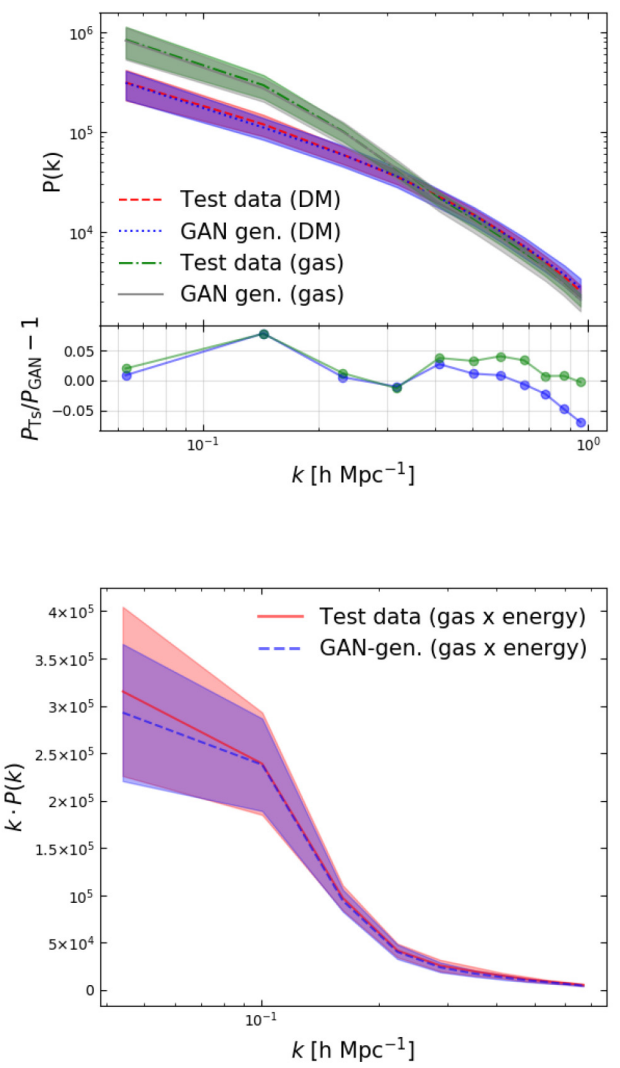

Figure 15. A selection of diagnostics to compare the test and the GAN-produced multicomponent cosmic web slices. Top left: The mean and the standard deviation of the power spectrum for 1000 randomly chosen slices for both data sets along with the corresponding relative difference between the data sets (green for $P_{\mathrm{Ts}}^{\mathrm{gas}} / P_{\mathrm{GAN}}^{\mathrm{gas}}-1$ and blue for $P_{\mathrm{Ts}}^{\mathrm{DM}} / P_{\mathrm{GAN}}^{\mathrm{DM}}-1$ ); top right: same as top left but with Gaussian smoothing applied; bottom left: the cross power spectrum calculated between 1000 randomly chosen dark matter and the corresponding gas cosmic web pairs for both the test and the GAN-produced data sets; bottom right: same as bottom left but for the gas-energy cross-power.

gas as well as the internal energy distributions on all scales as expected.

The Minkowski functional analysis (Fig. 16) revealed a generally good agreement between the two data sets, with significant differences appearing only in the boundary and the Euler characteristic Minkowski functionals for the energy cosmic web slices. This is somewhat surprising as the internal energy slices in general are significantly less complex on the smallest of scales when compared to the corresponding dark matter and gas data (see Fig. 3), hence we expected the GAN to easily learn to reproduce the named data set. However, we also found that the internal energy data and the corresponding Minkowski functionals are especially sensitive to adding any small-scale artificial noise. A more detailed Minkowski functional analysis is required to determine the reason for this divergence.

\subsection{Latent space interpolation results}

To perform the latent space interpolation procedure, we trained the GAN to produce cosmic web slices of two different redshifts along with weak lensing maps of different $\sigma_{8}$ values. Once trained, we produced a batch of outputs and in each case chose a pair of slices/maps corresponding to different redshifts or $\sigma_{8}$ values. Subsequently, we interpolated between the input vectors $Z_{1}$ and $Z_{2}$ corresponding to the outputs with different redshifts and $\sigma_{8}$ values (see Fig. 2).

Fig. 17 illustrates the results of the latent space interpolation procedure. In particular, it shows that the technique does indeed produce intermediate power spectra. However, the transition is not linear - the power spectra lines corresponding to equally spaced inputs (in the latent space) are not equally spaced in the power spectrum space. This is the case as the produced data samples can be described as points on a Riemannian manifold, which in general has curvature (see Appendix B for more details).

Figs 17 and 18 show the results of interpolating between cosmic web slices with redshifts $z=0.0$ and $z=1.0$ and weak lensing maps with $\sigma_{8}=0.436$ and $\sigma_{8}=0.814$. The interpolated samples are statistically realistic and the transition is nearly smooth.

An important part of the latent space interpolation procedure is being able to distinguish between the GAN-generated cosmic web slices and weak lensing maps of different redshifts, cosmologies, and modified gravity parameters. In this regard, we have used tested two machine learning algorithms: a convolutional neural network and gradient boosted decision trees. We initially used a three-layer convolutional neural network with 128 and 64 output filters of the convolution correspondingly with kernel size equal to $3 \times 3 \mathrm{px}$ and tanh activation functions. We found that the neural network approach has mostly failed to distinguish between the different data set classes reliably. After a thorough hyperparameter search we managed to reach accuracy of around 75 per cent, which was not good enough for the given task. We found that the gradient boosted decision tree algorithm (XGBoost; Chen \& Guestrin 2016) was fast and accurate when predicting the data set class. In particular, we reached 95 98 per cent accuracy (depending on the data set and hyperparameters used), when predicting the data set class of unseen test samples. 

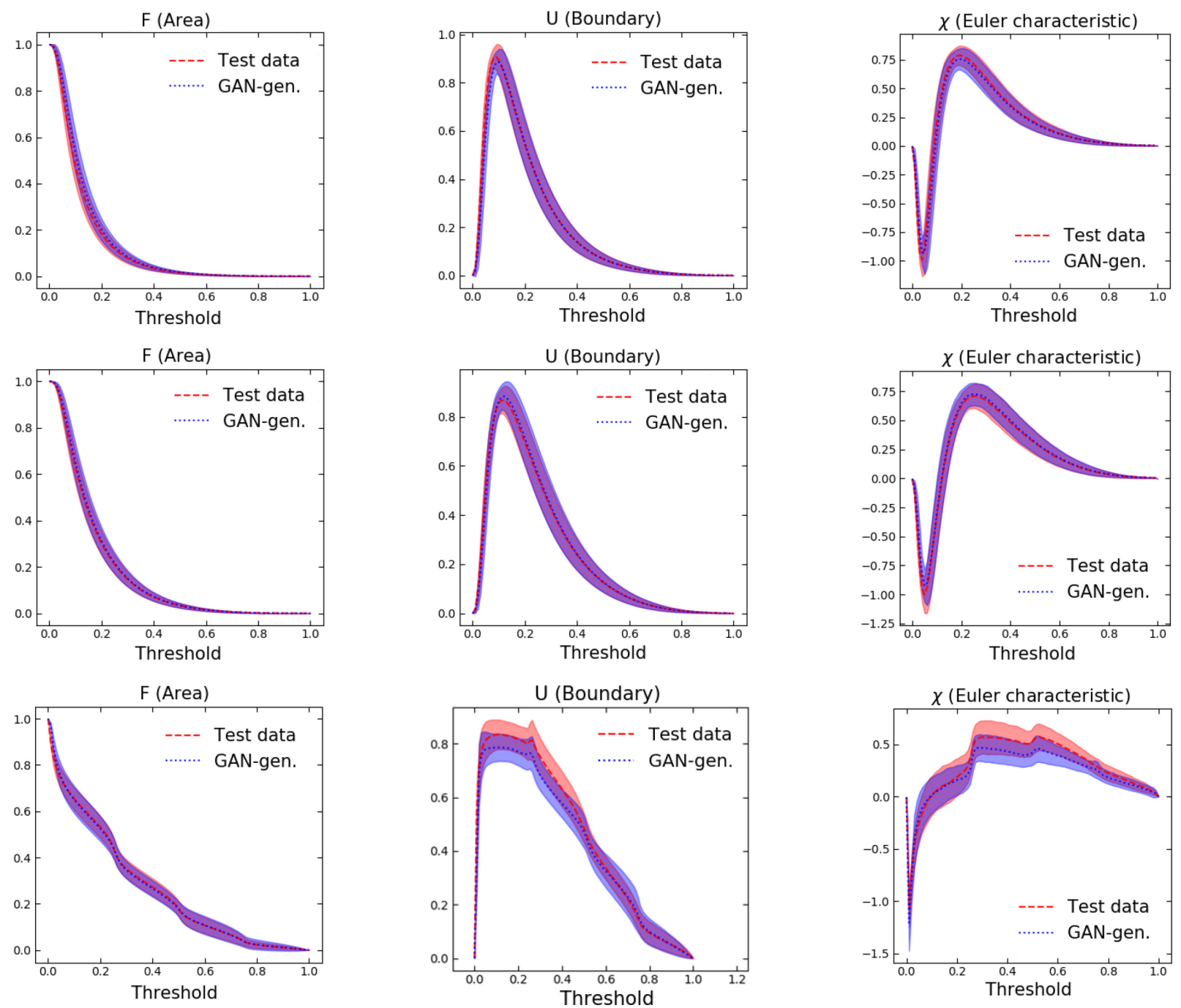

Figure 16. Results of the Minkowski functional analysis for the GAN trained on the DM, gas, and the internal energy data. Top row: Minkowski functionals for the DM cosmic web slices; middle row: Minkowski functionals for the gas overdensity slice data; bottom row: the corresponding Minkowski functionals for the internal energy data. In all cases Gaussian smoothing is applied.

Table 1 summarizes the parameters used when training the XGBoost algorithm.

Combining such a machine learning approach with a power spectrum analysis allowed us to distinguish between the different classes of the GAN-produced outputs reliably.

The latent space interpolation results illustrate a number of interesting features of GANs. First, the results illustrate that the GAN training procedure tightly encodes the various features discovered in our training data set in the high-dimensional latent space. By finding clusters in this latent space, corresponding to outputs of different redshifts or cosmology parameters, and linearly interpolating between them, we can produce outputs with intermediate values of the mentioned parameters. This allows us to indirectly control the outputs produced by the generator. In addition, as illustrated by Fig. 17, it is possible to discover vectors in the latent space that correspond to changes in redshift and $\sigma_{8}$. More generally, different vectors in the latent space correspond to various features of the data set, for instance the shapes of the filaments and the voids in the case of the cosmic web slices. This illustrates that the latent space interpolation technique is crucial for controlling the outputs of the GAN algorithm as well as accessing information about the important data set features learnt by the GAN during the training procedure. It is important to note, however, that these features are known to be entangled, such that sampling input points along some vector in the latent space inevitably introduces multiple changes to the generated outputs (see Section 6 for a further discussion of this issue).

\section{ANALYSIS AND CONCLUSIONS}

The main goal of this work was to investigate whether GANs can be used as a fast and efficient emulator capable of producing realistic and novel mock data. Likewise, we investigated the model feature space using the technique of latent space interpolation. Our results are encouraging, illustrating that GANs are indeed capable of producing realistic mock data sets. In addition, we have shown that GANs can be used to emulate dark matter, gas, and internal energy distribution data simultaneously. This is a key result as generating realistic gas distributions requires complex and computationally expensive hydrodynamical simulations. Hence, producing vast amounts of realistic multicomponent mock data quickly and efficiently will be of special importance in the context of upcoming observational surveys. 


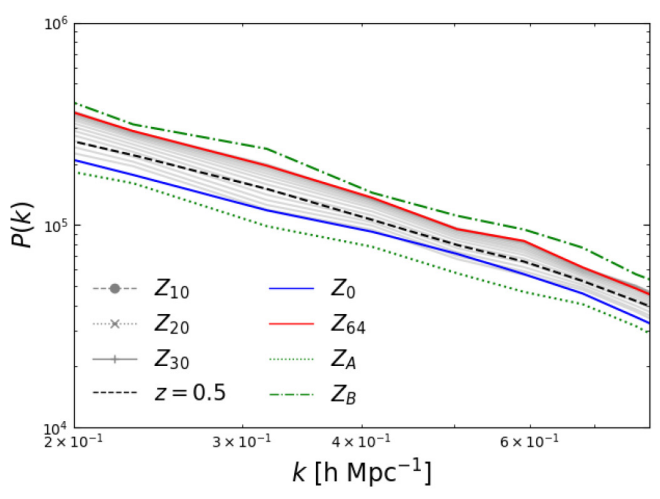

(a) $\mathrm{CW}$ slice redshift interpolation

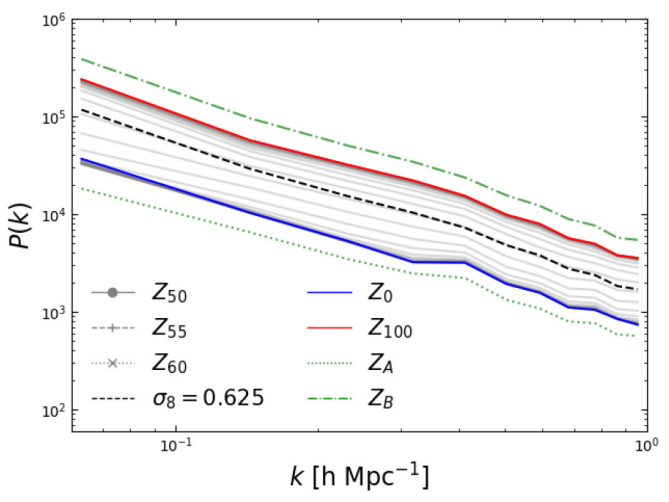

(b) WL $\sigma_{8}$ interpolation

Figure 17. The results of the linear latent space interpolation technique. Left: The matter power spectrum corresponding to a linear interpolation between two cosmic web slices of redshifts $z=0.0$ and $z=1.0$. The lines in grey are a selection of intermediate output slices generated by the procedure, while the black line corresponds to the mean value of the power spectrum calculated by choosing 100 random (test data) slices of redshift $z=0.5$. Right: Interpolating between two randomly chosen weak lensing maps with different values of $\sigma_{8}$. As before, the black line corresponds to the mean power spectrum produced from 100 random maps with $\sigma_{8}=0.625$. The grey lines were produced by sampling 64 and 100 latent space points correspondingly. Lines denoted by $Z_{A}$ and $Z_{B}$ indicate the power spectra resulting from interpolating beyond the $Z_{0}$ and $Z_{64} / Z_{100}$ points by an amount of $\mathrm{d} Z=Z_{2}-Z_{1}$.

\section{z interpolation:}
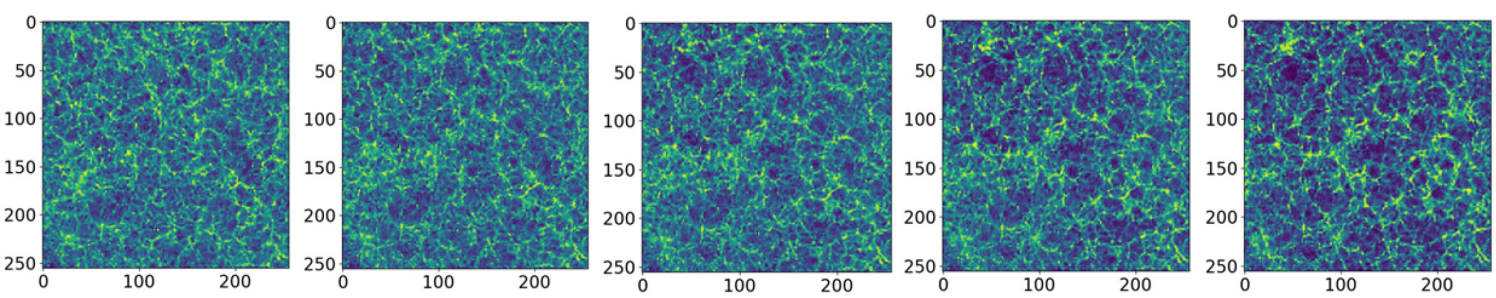

$\sigma_{8}$ interpolation:
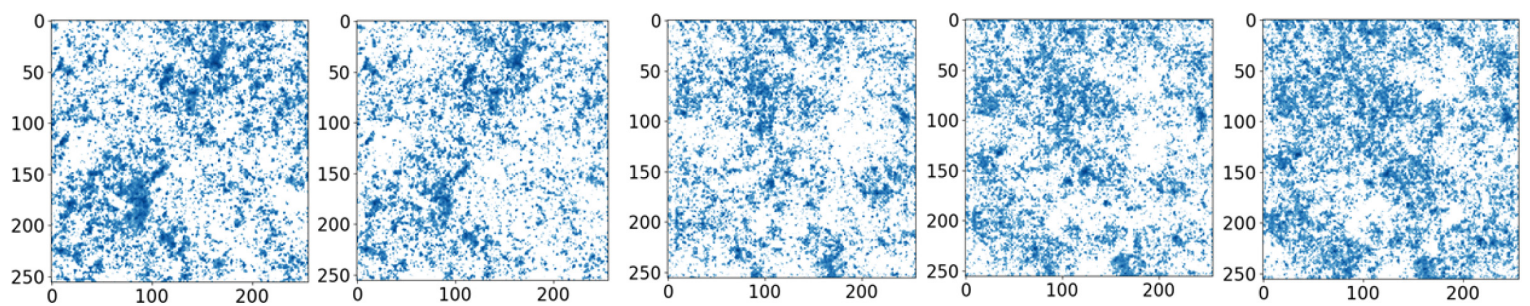

Figure 18. The results of the latent space interpolation procedure for cosmic web slices of redshifts $z=0.0$ (far right) and $z=1.0$ (far left) and weak lensing convergence maps of $\sigma_{8}^{1}=0.436$ (far left) and $\sigma_{8}^{1}=0.814$ (far right).

Table 1. The XGBoost parameters used for classifying the cosmic web slices with redshifts $z=\{0.0,1.0\}$ and the weak lensing maps with $\sigma_{8}=\{0.436$, $0.814\} . R_{\mathrm{L}}^{\mathrm{XGB}}$ refers to the learning rate and $D_{\max }$ is the maximum tree depth.

\begin{tabular}{lcccc}
\hline Parameter: & $R_{\mathrm{L}}^{\mathrm{XGB}}$ & $D_{\max }$ & Training step & Objective \\
\hline Value: & 0.08 & 2 & 0.3 & multi:softprob \\
\hline
\end{tabular}

The GAN-produced data in general cannot be distinguished from the training data set visually. In terms of the power spectrum analysis, the relative difference between the GAN-produced and the test data ranges between 1 and 20 percent depending on the data set and whether Gaussian smoothing was applied. The Minkowski functional analysis revealed a generally good agreement between the two data sets with an exception of the third Minkowski functional corresponding to curvature, which showed subtle differences for all studied data sets. In addition, greater differences were observed when training the GAN on data sets with multiple data classes. This is somewhat expected, as the training task becomes more difficult. In general, when training on data sets with multiple redshifts and cosmological parameters, our algorithm is capable of separating different data sets. It is important to emphasize, however, that this depends significantly on the data set used and on how different the cosmological parameters are. For data sets with significant overlap in terms of parameters of interest, an alternative approach might be required. In addition, it is important to note that the observed differences between the GAN-generated and the test data can be partially accounted for as a result of the small-scale noise in the emulated images. We found Gaussian smoothing with a $3 \times 3$ pixel kernel size to be effective in filtering away most of such noise. Finally, we note that the training data sets used in this work are smaller than those used in Rodríguez et al. (2018) and Mustafa et al. (2019), which, at least partially, accounts for the differences between our and their corresponding results. 
We also investigated a commonly used technique of latent space interpolation as a tool for investigating the feature space of the model. Interestingly, we found that such a procedure allows us to generate samples with intermediate redshift/cosmology $/ f_{R 0}$ parameter values, even if our model had not been explicitly trained on those particular values. In addition, the latent space interpolation procedure offers an indirect way of controlling the outputs of the GAN. However, it is important to point out some of the drawbacks of this procedure. Namely, as pointed out in machine learning literature, the latent space of a convolutional GAN is known to be entangled. In other words, moving in a different direction in the latent space necessarily causes multiple changes to the outputs of the GAN. As a concrete example, finding a latent space line that induces a change in redshift of a given output necessarily also introduces other subtle changes to the output (e.g. the depth of the voids or the distribution of the filaments). So if we take a random output of redshift $z=1.0$ and perform the linear interpolation procedure to obtain a cosmic web slice of $z=0.0$, the obtained slice will correspond to a realistic but different distribution of the required redshift. This is a drawback as in an ideal case we would love to have full control of individual parameters, while not affecting other independent features of a data set. There are, however, other generative models discussed in the literature that allow such manipulation of the latent space. Namely, the $\beta$-VAE and the InfoGAN algorithms allow encoding features into the latent space in a special way that allows full control of individual key parameters without affecting the other features of the data set (latent space disentanglement; Chen et al. 2016; Higgins et al. 2017; Burgess et al. 2018).

As illustrated by our results, the technique of latent space interpolation can be used to indirectly control the outputs of the GAN algorithm. A significant disadvantage of such approach is that it is based on the ability to find certain vectors in the latent space, which, given its high dimensionality, is not trivial. In addition, it is difficult to tell apart outputs corresponding to different cosmological parameters (hence the need for an extra machine learning classifier). These issues, however, can be tackled by directly specifying the cosmological parameters as an extra input vector alongside the usual latent space input. Such an approach is applied in the recent GAN architectures such as the mentioned conditional GAN algorithm (Mirza \& Osindero 2014; Perraudin et al. 2020). The conditional GAN architecture also makes it easier to interpolate between the outputs corresponding to different cosmological parameters. None the less, even though the code used in this work is not the ideal tool for directly controlling the GAN outputs, it holds multiple advantages when training on a single-parameter data sets. Namely, our opensource code (see the data availability section) is well tested and easily customizable. In addition, as illustrated by our Illustris data set results, our code allows training multiple data components (e.g. dark matter, gas, and energy distribution data) simultaneously with no difficulty. This makes it particularly easy to apply the techniques laid out in this work on other kinds of data sets. Lastly, it should be noted that the latent space interpolation techniques presented here are architecture-independent and thus can be easily integrated into newer GAN architectures.

Another important topic to discuss is the problem of mode collapse. As is widely discussed in the literature, the generator neural network is prone to getting stuck in producing a very small subsample of realistic mock data points that fool the discriminator neural network. Resolving mode collapse is an important open problem in the field of deep learning, with a variety of known strategies ranging from choosing a particular GAN architecture, to altering the training procedure or the cost function (Srivastava et al. 2017; Yicheng \&
Hong 2019). Mode collapse was encountered multiple times in our training procedure as well. As a rule of thumb, we found that reducing the learning rate parameter had the biggest effect towards resolving mode collapse for all studied data sets. Learning rates around the values of $3 \times 10^{-5}$ for the cosmic web data and $9 \times 10^{-6}$ for the weak lensing maps were found to be the most effective in avoiding any mode collapse.

As we have shown, GANs can be used to generate novel 2D data efficiently. A natural question to ask is whether this also applies to 3D data. As an example, an analogous emulator capable of generating 3D cosmic web data, such as that produced by stateof-the-art hydrodynamic and DM-only simulations would be very useful. In principle there is no limit on the dimensionality of the data used for training a GAN, however, in practice, going from 2D to $3 \mathrm{D}$ data leads to a significant increase of the generator and the discriminator networks. In addition, in the case of 3D cosmic web data, forming a big enough training data set would become an issue, as running thousands of simulations would be required. However, as previously mentioned, there are sophisticated ways of emulating 3D cosmic web data as shown in Perraudin et al. (2019), where a system of GANs is used to upscale small resolution comic web cubes to full size simulation boxes. Note that the techniques introduced in this work (e.g. latent space interpolation) can be readily combined with the mentioned 3D approach.

A number of other interesting directions can be explored in future work. Namely, a more detailed investigation into the Riemannian geometry of GANs could lead to a better understanding of the feature space of the algorithm. Finally, many other data sets could be explored. With upcoming surveys, such as Euclid, generating mock galaxy and galaxy cluster data quickly and efficiently is of special interest. A GAN could be used to generate galaxies with realistic intrinsic alignments, density distributions, and other properties. Similarly, GANs could be used to quickly emulate realistic galaxy cluster density distributions at a fraction of the computational cost required to run full hydrodynamic simulations.

To conclude, GANs offer an entirely new approach for cosmological data emulation. Such a game theory based approach has been demonstrated to offer a quick and efficient way of producing novel data for a low computational cost. As we have shown in this work, the trade-off for this is a 1-20 per cent difference in the power spectrum, which can be satisfactory or not depending on what application such an emulator is used for. Even though a number of questions remain to be answered regarding the stability of the training procedure and training on higher dimensional data, GANs will undoubtedly be a useful tool for emulating cosmological data in the era of modern $\mathrm{N}$-body simulations and precision cosmology.

\section{ACKNOWLEDGEMENTS}

We thank the Columbia Lensing group (http://columbialensing.org) for making their suite of simulated maps available, and National Science Foundation (NSF) for supporting the creation of those maps through grant AST-1210877 and XSEDE allocation AST-140041. This work would also not have been possible without the access to the HPC Sciama facilities at the Institute of Cosmology and Gravitation and the support by the IT staff. We also thank Adam Amara for the guidance and the useful discussions. Finally, we thank Minas Karamanis for the help with the statistical analysis. AT is supported by the Science \& Technology Facilities Council (STFC) through the DISCnet Centre for Doctoral Training funding. The Google Cloud computational resources were funded by the Research and Innovation Funding by the University of Portsmouth. 
KK has received funding from the European Research Council (ERC) under the European Union's Horizon 2020 research and innovation programme (grant agreement No. 646702 'CosTesGrav'). DB and KK are also supported by the UK STFC ST/S000550/1.

\section{DATA AVAILABILITY}

The key data sets generated in this work as well as the main analysis scripts are available at following GitHub repository: https://github.com/AndriusT/cw_wl_GAN. The link also contains detailed instructions on how to produce the data samples from the publicly available Illustris data. The full Illustris data sets can be found at: https://www.illustris-project.org/data/.

The used weak lensing data sets with different cosmological parameters can be accessed at: http://columbialensing.org/.

\section{REFERENCES}

Arjovsky M., Chintala S., Bottou L., 2017, preprint (arXiv:1701.07875)

Burgess C. P., Higgins I., Pal A., Matthey L., Watters N., Desjardins G., Lerchner A., 2018, preprint (arXiv:1804.03599)

Chen T., Guestrin C., 2016, Proceedings of the 22nd ACM SIGKDD International Conference on Knowledge Discovery and Data Mining. KDD '16. Association for Computing Machinery, New York, NY, p. 785

Chen X., Duan Y., Houthooft R., Schulman J., Sutskever I., Abbeel P., 2016, preprint (arXiv:1606.03657)

Columbia Lensing, 2020, Columbia Lensing Group Data Products, http://co lumbialensing.org (accessed 2020-01-24)

Dietrich J. P., Hartlap J., 2010, MNRAS, 402, 1049

Farnia F., Ozdaglar A., 2020, preprint (arXiv:2002.09124)

Fukushima K., 1980, Biol. Cybern., 36, 193

Goodfellow I. J., Pouget-Abadie J., Mirza M., Xu B., Warde-Farley D., Ozair S., Courville A., Bengio Y., 2014, preprint (arXiv:1406.2661)

Gupta A., Matilla J. M. Z., Hsu D., Haiman Z., 2018, Phys. Rev. D, 97, 103515

Hand N., Feng Y., Beutler F., Li Y., Modi C., Seljak U., Slepian Z., 2018, ApJ, 156, 160

Hauser M., Ray A., 2017, in Guyon I., Luxburg U. V., Bengio S., Wallach H., Fergus R., Vishwanathan S., Garnett R., eds, Advances in Neural Information Processing Systems 30. Curran Associates, Inc., Red Hook, NY 12571, USA, p. 2807

He S., Li Y., Feng Y., Ho S., Ravanbakhsh S., Chen W., Póczos B., 2019, Proc. Natl. Acad. Sci., 116, 13825

Higgins I., Matthey L., Pal A., Burgess C., Glorot X., Botvinick M. M., Mohamed S., Lerchner A., 2017, ICLR 2017

Howlett C., Manera M., Percival W., 2015, Astron. Comput., 12, 109

Ioffe S., Szegedy C., 2015, preprint (arXiv:1502.03167)

Kingma D. P., Welling M., 2013, preprint (arXiv:1312.6114)

Knabenhans M. et al., 2019, MNRAS, 484, 5509

Kodi Ramanah D., Charnock T., Villaescusa-Navarro F., Wandelt B. D., 2020, MNRAS, 495, 4227

Kwan J., Heitmann K., Habib S., Padmanabhan N., Lawrence E., Finkel H., Frontiere N., Pope A., 2015, ApJ, 810, 35

Lacoste A., Luccioni A., Schmidt V., Dandres T., 2019, preprint (arXiv:1910.09700)

Lecun Y., Bottou L., Bengio Y., Haffner P., 1998, Proc. IEEE, 86, 2278

Li B., Koyama K., 2019, Modified Gravity: Progresses and Outlook of Theories, Numerical Techniques and Observational Tests. World Scientific Publishing Company Pte Limited, Singapore

Li S.-Y., Li Y.-L., Zhang T.-J., 2019, Res. Astron. Astrophys., 19, 137

Ling C., Wang Q., Li R., Li B., Wang J., Gao L., 2015, Phys. Rev. D, 92, 064024

Linnainmaa S., 1976, BIT Numer. Math., 16, 146
Maas A. L., Hannun A. Y., Ng A. Y., 2013, in ICML Workshop on Deep Learning for Audio, Speech and Language Processing

Mantz H., Jacobs K., Mecke K., 2008, J. Stat. Mech.: Theory Exp., 2008, P12015

Marques G. A., Liu J., Zorrilla Matilla J. M., Haiman Z., Bernui A., Novaes C. P., 2019, J. Cosmol. Astropart. Phys., 2019, 019

Mawdsley B. et al., 2020, MNRAS, 493, 5662

Merten J., Giocoli C., Baldi M., Meneghetti M., Peel A., Lalande F., Starck J.-L., Pettorino V., 2019, MNRAS, 487, 104

minkfncts2d Python package, 2020, https://github.com/cefarix/minkfncts2d (accessed 2020-03-09)

Mirza M., Osindero S., 2014, preprint (arXiv:1411.1784)

Mishra A., Reddy P., Nigam R., 2019, preprint (arXiv:1908.04682)

Mustafa M., Bard D., Bhimji W., Lukić Z., Al-Rfou R., Kratochvil J. M., 2019, Comput. Astrophys. Cosmol., 6, 1

Mustafa M., Bard D., Bhimji W., Lukić Z., Al-Rfou R., Kratochvil J. M., 2020, cosmoGAN. https://github.com/MustafaMustafa/cosmoGAN (accessed 2020-01-24)

Nair V., Hinton G. E., 2010, ICML'10. Omnipress, Madison, WI, p. 807

Nelson D. et al., 2015, Astron. Comput., 13, 12

Ntampaka M. et al., 2019, BAAS, 51, 14

Perraudin N., Srivastava A., Lucchi A., Kacprzak T., Hofmann T., Réfrégier A., 2019, Comput. Astrophys. Cosmol., 6, 5

Perraudin N., Marcon S., Lucchi A., Kacprzak T., 2020, 33rd Conference on Neural Information Processing Systems (NeurIPS), preprint (arXiv:2004.08139)

Petri A., Haiman Z., Hui L., May M., Kratochvil J. M., 2013, Phys. Rev. D, 88,123002

Radford A., Metz L., Chintala S., 2015, preprint (arXiv:1511.06434)

Rodríguez A. C., Kacprzak T., Lucchi A., Amara A., Sgier R., Fluri J., Hofmann T., Réfrégier A., 2018, Comput. Astrophys. Cosmol., 5,4

Rumelhart D., Hinton G. E., Williams R. J., 1986, Nature, 323, 533

Scoccimarro R., Hui L., Manera M., Chan K. C., 2012, Phys. Rev. D, 85, 083002

Shao H., Kumar A., Fletcher P. T., 2017, preprint (arXiv:1711.08014)

Smith M. J., Geach J. E., 2019, MNRAS, 490, 4985

Springel V., 2005, MNRAS, 364, 1105

Srivastava A., Valkov L., Russell C., Gutmann M. U., Sutton C., 2017, preprint (arXiv:1705.07761)

Tassev S., Zaldarriaga M., Eisenstein D. J., 2013, J. Cosmol. Astropart. Phys., 2013, 036

Ullmo M., Decelle A., Aghanim N., 2020, Astronomy and Astrophysics, 651, A46

Vogelsberger M. et al., 2014, Nature, 509, 177

Winther H. A., 2020, MG-PICOLA. https://github.com/HAWinther/MG-PI COLA-PUBLIC (accessed 2020-03-26)

Winther H. A., Koyama K., Manera M., Wright B. S., Zhao G.-B., 2017, J. Cosmol. Astropart. Phys., 2017, 006

Winther H. A., Casas S., Baldi M., Koyama K., Li B., Lombriser L., Zhao G.-B., 2019, Phys. Rev. D, 100, 123540

Yicheng H., 2019, preprint (arXiv:1910.04636)

Zorrilla Matilla J. M., Haiman Z., Hsu D., Gupta A., Petri A., 2016, Phys. Rev. D, 94, 083506

\section{APPENDIX A: SAMPLES OF THE GAN-PRODUCED DATA}

This section contains a selection of GAN-produced samples for visual inspection. Fig. A1 contains randomly selected weak lensing convergence maps produced by the GAN algorithm (these are the samples described in Sections 5.2 and 5.3).

Fig. A2 shows a selection of randomly selected cosmic web 2D slices for two different redshifts. Both the training data and the produced slices have been Gaussian-smoothed. 
Training data:
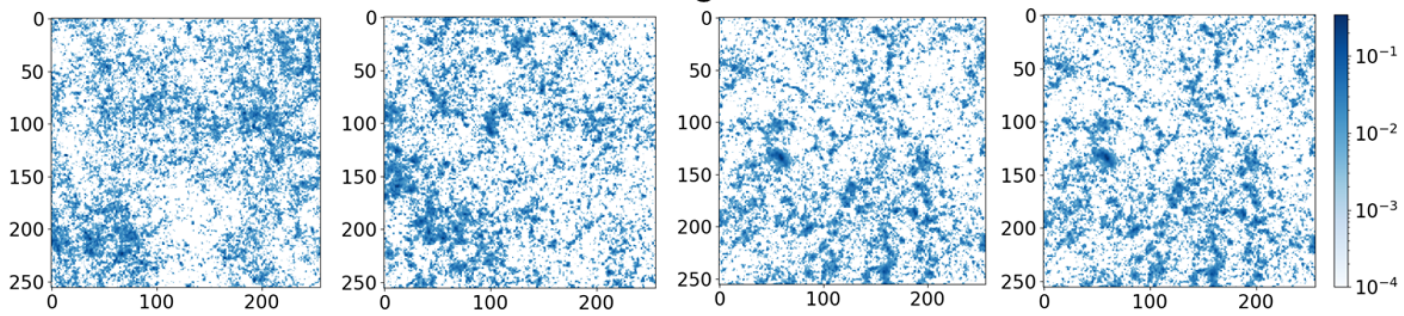

GAN-generated:
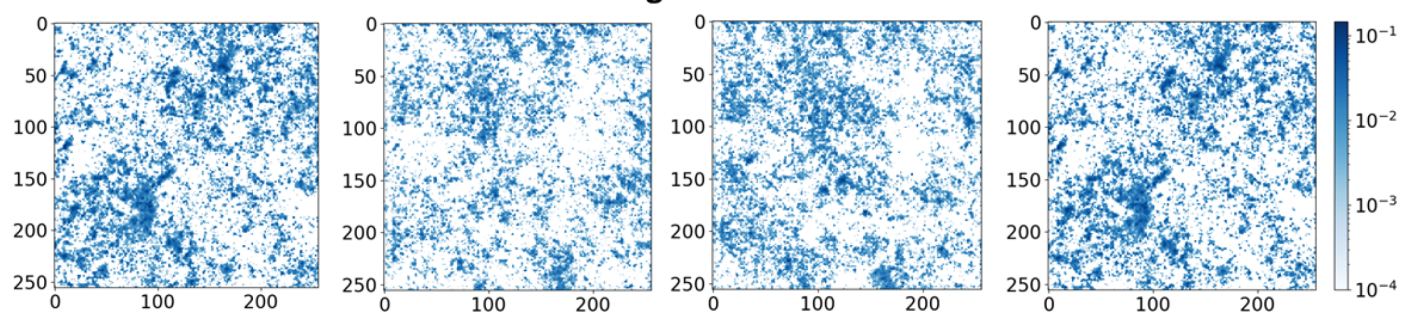

Figure A1. A comparison of four randomly selected weak lensing convergence maps. The colours are log-normalized to emphasize the main features and to allow a direct comparison with the previous results in the literature.

Training data:
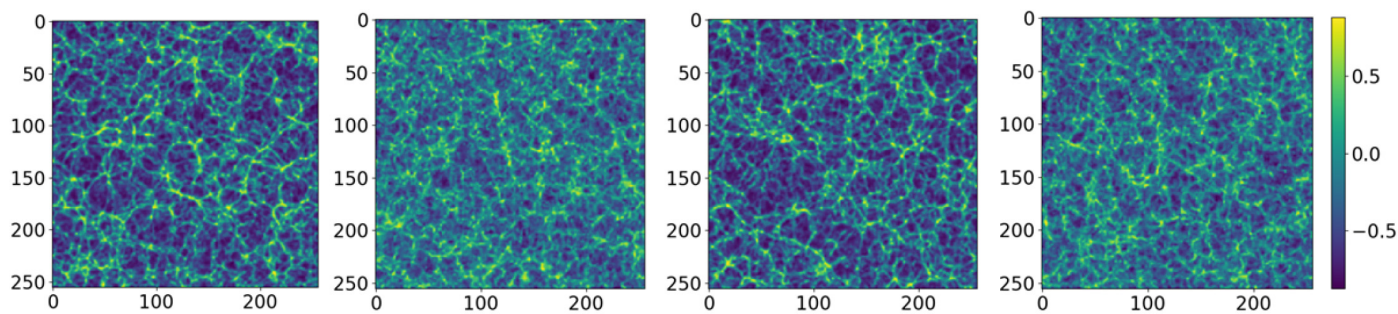

GAN-generated:
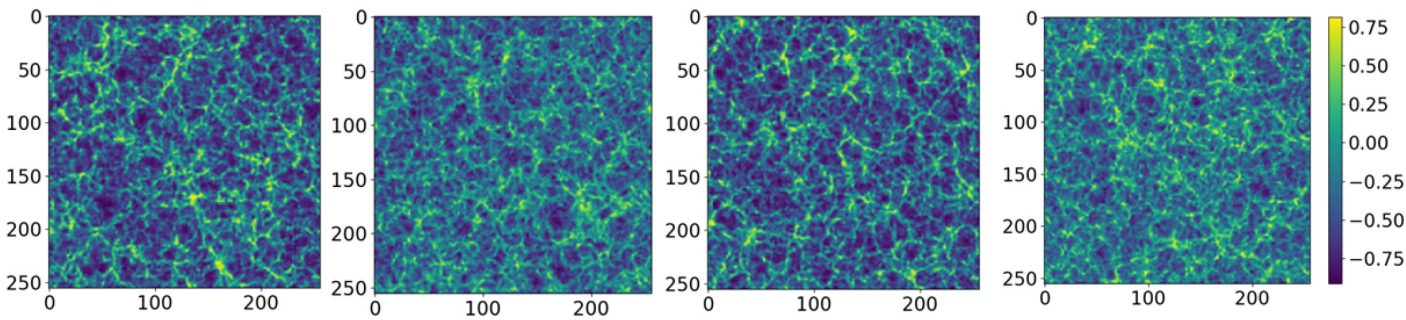

Figure A2. A comparison of four randomly selected cosmic web slices. Columns 1 and 3 correspond to redshift 0.0 while columns 2 and 4 are redshift 1.0 .

\section{APPENDIX B: RIEMANNIAN GEOMETRY OF THE GAN ALGORITHM}

Recently various connections between GANs and Riemannian geometry have been explored in the machine learning literature. Such connections are important to explore, not only for the sake of curiosity, but also because they allow us to describe GANs and their optimization procedure in a language more familiar to physicists. A Riemannian geometry description of GANs is also powerful when exploring the latent space of a trained generator neural network and the outputs that it produces. Finally, a differential geometry description could shine some light on the connections between generative models and information geometry, which is a wellestablished field and could offer some new insights into training and analysing the outputs of such models.
Recent work in Shao et al. (2017) proposes treating the trained generator neural network as a mapping from a lower dimensional latent space $Z$ to the higher dimensional data space $X: G_{\phi}: Z \rightarrow X$ (see Fig. B1). More specifically, the generator $G_{\phi}(Z)$ maps the latent space vectors of size $n$ (in our case $n=256$ or 64) to a manifold $M$ of dimensionality $m(256 \times 256$, i.e. the number of pixels in the output images). Manifold $M$ here simply refers to a subset of the data space (all possible combinations of pixel values), which correspond to realistic images of weak lensing / cosmic web slices. The existence of such a manifold is postulated by the manifold hypothesis in deep learning, which states that high-dimensional data can be encoded on a manifold of a much lower dimension.

Hence if we treat the generator neural network $G_{\phi}$ as a mapping for the latent space to the data space manifold, one can naturally define an induced metric $g$, which is simply a product of the Jacobian and 


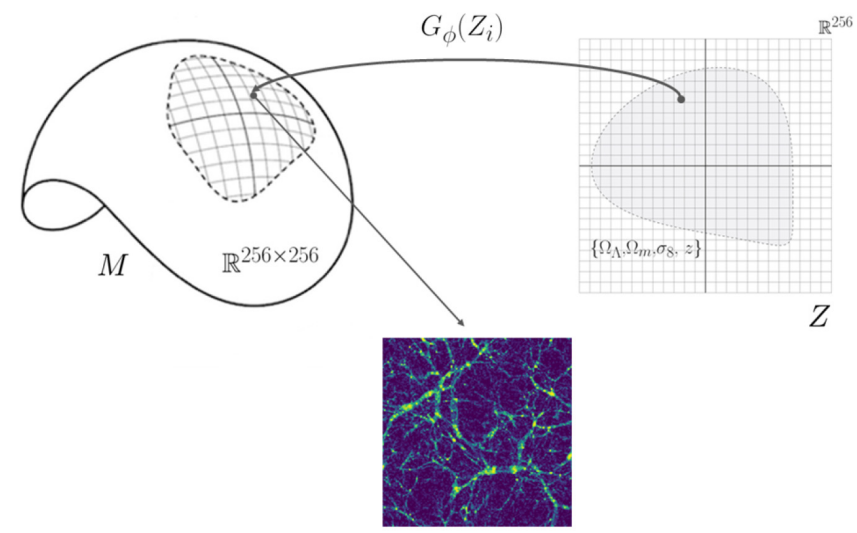

Figure B1. Riemannian geometry of generative adversarial networks. The generator $G_{\phi}\left(Z_{i}\right)$ can be treated as a mapping from the lower dimensional Euclidean latent space $Z$ (corresponding to the random noise input) to the high-dimensional data (pixel) space $M$ (in general non-Euclidean). Each point on $M$ corresponds to a weak lensing map (or a cosmic web slice).

the transposed Jacobian:

$g=J(Z)^{T} J(Z)$

The Jacobian in our case simply refers to the partial derivative of each output value w.r.t. to each input value, i.e.:

$J=\left[\begin{array}{cccc}\frac{\partial X^{1}}{\partial Z^{1}} & \frac{\partial X^{1}}{\partial Z^{2}} & \cdots & \frac{\partial X^{1}}{\partial Z^{n}} \\ \vdots & \vdots & \ddots & \\ \frac{\partial X^{m}}{\partial Z^{1}} & \frac{\partial X^{m}}{\partial Z^{2}} & \cdots & \frac{\partial X^{m}}{\partial Z^{n}}\end{array}\right]$

Once a metric is defined, we can use the usual tools to describe geodesics on the manifold $M$. For instance, we can define a curve $\kappa$ between two points $a$ and $b$ in the latent space $Z$ parametrized by some parameter $t$. Using the mapping $G_{\phi}$, the corresponding curve on the manifold $M$ is then: $G_{\phi}(\kappa(t)) \in M$. To find a curve that corresponds to a geodesic on the manifold, one has to solve the Euler-Lagrange equation:

$\frac{\mathrm{d}^{2} \kappa^{\alpha}}{\mathrm{d} t^{2}}=-\Gamma_{\beta \gamma}^{\alpha} \frac{\mathrm{d} \kappa^{\beta}}{\mathrm{d} t} \frac{\mathrm{d} \kappa^{\gamma}}{\mathrm{d} t}$,

where $\Gamma$ is the usual Christoffel symbol, given by

$\Gamma_{\beta \gamma}^{\alpha}=\frac{1}{2} g^{\alpha \delta}\left(\frac{\partial g_{\delta \beta}}{\partial X^{\gamma}}+\frac{\partial g_{\delta \gamma}}{\partial X^{\alpha}}-\frac{\partial g_{\alpha \beta}}{\partial X^{\delta}}\right)$.

As discussed in Shao et al. (2017), geodesics between points on the manifold are of special importance as they give the smoothest possible transition between multiple outputs. One of the main findings in Shao et al. (2017) was that the Riemannian curvature of the manifold corresponding to the their data was surprisingly small and, hence, linear interpolation produced realistic results comparable to the results produced by calculating a geodesic curve between outputs. In our work, we also found that linear interpolation generally produced realistic results. However, to ensure that the outputs produced via the latent space interpolation are indeed realistic, one would have to interpolate on a curve in the latent space (corresponding to the geodesic connecting the needed outputs on the data manifold $M$ ) rather than a line.

Another important connection to Riemannian geometry comes in the context of the discriminator neural network. The discriminator can be viewed as a mapping from the data manifold to a probability manifold $P$, where each point on the manifold corresponds to the probability of a given data sample being real (i.e. belonging to the training data set). Such a manifold looks remarkably similar to the statistical manifolds studied in the field of information geometry. Insights from information geometry have a long tradition of being used in neural network optimization (e.g. Hauser \& Ray 2017). Exploring such connections could lead to deeper insights into the GAN training process, which we plan to explore in future work.

\section{APPENDIX C: GENERATOR AND THE DISCRIMINATOR ARCHITECTURE}

The architecture used when training the GAN algorithm is summarized in Tables $\mathrm{C} 1$ and $\mathrm{C} 2$.

Table C1. The architecture of the generator neural network. TConv corresponds to the transposed convolutional layer with stride $=2$ (and the kernel size given by the shown numerical values). ReLU corresponds to the rectified linear unit activation function. $L R e L U$ stands for the leaky rectified linear unit activation function with the leakiness parameter $=0.2$.

\begin{tabular}{lccc}
\hline & Activ. & Output shape & Params. \\
\hline Latent & - & 64 & - \\
Dense & - & $512 \times 16 \times 16$ & $8.5 \mathrm{M}$ \\
BatchNorm & ReLU & $512 \times 16 \times 16$ & 1024 \\
TConv $5 \times 5$ & - & $256 \times 32 \times 32$ & $3.3 \mathrm{M}$ \\
BatchNorm & ReLU & $256 \times 32 \times 32$ & 512 \\
TConv $5 \times 5$ & - & $128 \times 64 \times 64$ & $819 \mathrm{~K}$ \\
BatchNorm & ReLU & $128 \times 64 \times 64$ & 256 \\
TConv $5 \times 5$ & - & $64 \times 128 \times 128$ & $205 \mathrm{~K}$ \\
BatchNorm & ReLU & $64 \times 128 \times 128$ & 128 \\
TConv $5 \times 5$ & Tanh & $1 \times 256 \times 256$ & 1601 \\
Total trainable parameters & & $\mathbf{1 2 . 3 M}$ \\
\hline
\end{tabular}

Table C2. The architecture of the discriminator neural network.

\begin{tabular}{lccc}
\hline & Activ. & Output shape & Params. \\
\hline Input map & - & $1 \times 256 \times 256$ & - \\
Conv $5 \times 5$ & LReLU & $64 \times 128 \times 128$ & 1664 \\
Conv $5 \times 5$ & - & $128 \times 64 \times 64$ & $205 \mathrm{~K}$ \\
BatchNorm & LReLU & $128 \times 64 \times 64$ & 256 \\
Conv $5 \times 5$ & - & $256 \times 32 \times 32$ & $819 \mathrm{~K}$ \\
BatchNorm & LReLU & $256 \times 32 \times 32$ & 512 \\
Conv $5 \times 5$ & - & $512 \times 16 \times 16$ & $3.3 \mathrm{M}$ \\
BatchNorm & LReLU & $512 \times 16 \times 16$ & 1024 \\
Linear & Sigmoid & 1 & $131 \mathrm{~K}$ \\
Total trainable parameters & & $\mathbf{4 . 4 M}$ \\
\hline
\end{tabular}




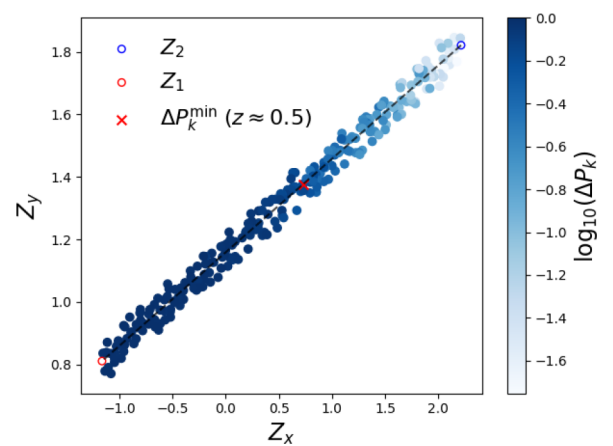

Figure D1. The visualization of the latent space clustering. The points are coloured based on equation (D1) with the mean power spectrum of 1000 randomly chosen cosmic web slices with redshift $z=0.0$ as the base power spectrum. The axes correspond to two randomly chosen axes out of the 256 dimensions in the latent space. The red $\mathrm{x}$ marks the point that produces the power spectrum that is the closest to the mean power spectrum of 1000 cosmic web slices with $z=0.5$ (with the mean difference of 1.4 per cent).

\section{APPENDIX D: LATENT SPACE CLUSTERING}

To investigate the structure of the latent space, two random latent space points were selected corresponding to two cosmic web slices with redshifts $z=\{0.0,1.0\}$ and weak lensing maps with $\sigma_{8}=$ $\{0.436,0.814\}$. Then the line connecting the two points was found and a number of points (close to the line but otherwise randomly distributed) were sampled. For each point, a corresponding output was produced and the power spectrum was calculated. For each point, the resulting power spectrum was compared against a base power spectrum (corresponding to $z=0.0$ and $\sigma_{8}=0.436$ ). The comparison was done by calculating the following quantity:

$\Delta P_{k}=\sum_{i} \mid \log _{10}\left(\mathrm{P}\left(\mathrm{k}_{\mathrm{i}}\right)\right)-\log _{10}\left(\mathrm{P}^{\mathrm{B}}\left(\mathrm{k}_{\mathrm{i}}\right) \mid\right.$,

with $P^{B}$ as the base power spectrum. The mentioned statistic was then rescaled in the range of $[0.0,1.0]$ and used to colour the points

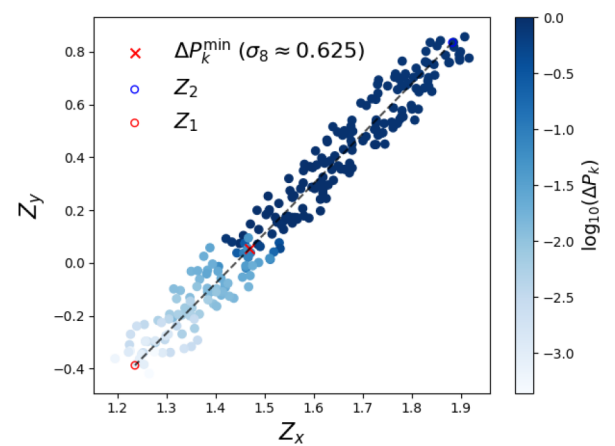

Figure D2. Same as Fig. D1 except for the weak lensing maps with $\sigma_{8}$ $=\{0.436,0.814\}$. The mean power spectrum of 1000 maps with $\sigma_{8}=0.436$ is used as the base power spectrum for equation (D1). The red $\mathrm{x}$ marks the point with the power spectrum closest to the mean power spectrum due to 1000 maps with $\sigma_{8}=0.625$ (with 2.7 per cent difference).

in Fig. D1. Note that the dimensionality of the latent space is too high to be fully visualized, hence a $2 \mathrm{D}$ projection was plotted instead (i.e. only 2 of the 256 dimensions were used).

Figs D1 and D2 illustrate some of the key properties of the latent space of the GAN algorithm. In particular, as expected, points that are nearby in latent space, produce outputs with similar power spectrum. Note that most of the plotted points produce outputs that have power spectra corresponding to either $z=0.0$ or $z=$ 1.0 or correspondingly to the two $\sigma_{8}$ values for the weak lensing maps. In addition, one can see that the transition between these two regimes is not entirely continuous (this depends partially on the chosen activation functions). None the less, in both cases there exists a cluster of points, near the limit between the two regimes, which produces power spectra nearly identical to $z=0.5$ and $\sigma_{8}=0.436$. One can always generate more points by zooming in that region in the latent space and sampling extra points.

This paper has been typeset from a $\mathrm{T}_{\mathrm{E}} \mathrm{X} / \mathrm{L} \mathrm{T} \mathrm{E} \mathrm{X}$ file prepared by the author. 